SINP-2002-7/691

\title{
Dyonic Black Holes with String-Loop Corrections
}

\author{
Mikhail Z. Iofa ${ }^{1}$ \\ Skobeltsyn Institute of Nuclear Physics \\ Moscow State University \\ Moscow 119992, Russia \\ November 21, 2018
}

\begin{abstract}
In heterotic string theory compactified to four dimensions with $\mathrm{N}=2$ supersymmetry, string-loop corrections to the universal sector of the low-energy effective action are studied. Within the framework of $\mathrm{N}=2$ supersymmetric formulation of the theory, in the first order in string coupling constant, we solve the system of the loop-corrected Maxwell and Killing spinor equations. Taking as the in-put the tree-level dyonic black hole solution, we calculate string-loop corrections to the string tree-level metric and moduli of dyonic black hole.
\end{abstract}

PACS: 04.70.Dy,04.50.+h,11.25.Db,11.25.Mj

Keywords: string theory, black holes, $\mathrm{N}=2$ supergravity

\footnotetext{
${ }^{1}$ Skobeltsyn Institute of Nuclear Physics, Moscow State University, Moscow 119992, Russia, e-mail: iofa@theory.sinp.msu.ru
} 


\section{Introduction}

At present, string theory is considered the best candidate for a fundamental theory that would be a consistent quantum theory of gravity unified with the other interactions [1]. In particular, in string theory a large number of classical solutions with horizons was found $[2,3]$ and refs. therein. In this setting, we meet a fundamental problem of understanding how the intrinsically stringy quantum effects modify the Einstein gravity and classical black-hole solutions of equations of motion.

In perturbative approach to string theory, the amplitudes are calculated as a sum of contributions from the string world sheets of different genera (for example, [1, 4]). The effective action, except for gravity and matter, contains a number of fields inherent to string theory, such as tensor fields, dilaton and other moduli. The string tree-level effective action of the light modes receives both $\alpha^{\prime}$ and string-loop corrections from higher genera (see, for example, the review [5]). Thus, the standard Einstein gravity and black-hole solutions considered within the framework of superstring theory are also modified by the string-loop corrections.

In this paper, we study the string-loop corrections to the tree-level black hole dyonic solution of the equations of motion of the $N=2$ supersymmetric $4 D$ theory obtained by a suitable compactification of the heterotic string theory. In distinction to the systems with $N=4$ supersymmetry, where the loop corrections to the two-derivative terms vanish, and the models with $N=1$ supersymmetry, where the amplitudes receive the infinite number of loop corrections [6], in models with $N=2$ supersymmetry loop corrections to the relevant objects vanish beyond one loop, which makes the problem of their account more or less tractable cf. $[7,8,9]$ and refs. therein.

Calculation of the string-loop corrections to the two-derivative terms of the bosonic part of the $N=2$ supersymmetric heterotic string tree-level effective action from the world sheets of torus topology in the path-integral approach shows that the Einstein term receives no correction, while the gauge couplings and the moduli part of the action are modified cf. [9]. Instead of performing rather complicated explicit path integral calculations to different terms of the action, following the usual practice, we make use of the general approach to $N=2$ locally supersymmetric theories [10] and refs. therein. Tree-level heterotic string effective action can be obtained from the prepotential of the $N=2$ supersymmetric $S T U$ model $[11,12,13]$. Loop corrections to the tree-level prepotential of the $N=2$ supersymmetric heterotic string effective action vanish beyond the one loop. Using the loop-corrected prepotential, it is possible to calculate the loop corrections to various terms of the effective action in a universal way.

To be concrete, we consider the effective action of the heterotic string theory compactified on the manifold $K 3 \times T^{2}$ or on a suitable orbifold yielding $N=2$ local supersymmetry in $4 D$.

To obtain classical solutions of supersymmetric theory, it is possible either to solve the second-order equations of motion, or Killing spinor equations which are conditions that supersymmetry variations of the fermionic components of the superfieldes to vanish. In this paper, to calculate the loop corrections to classical solutions, we follow the second way, solving the system of Killing spinor and Maxwell equations (cf. [14] and [15] where the loop corrections to magnetic black hole were calculated by solving the second-order equations equations of motion and Killing spinor equations). 
We consider the universal sector of the theory which contains gravity, the moduli connected with the metric $G_{m n}, m, n=1,2$ and the antisymmetric tensor $B_{12}$ of the two-torus $T^{2}$, and four vector fields which are expressed through the mixed internal-space-time components $G_{m \nu}$ and $B_{m \nu}$ of the metric and antisymmetric tensor and study dyonic solution of $N=2$ supersymmetric $4 D$ theory with two electric and two magnetic charges.

In the first order in string coupling constant $\epsilon=e^{\phi_{\infty}}$, where $\phi_{\infty}$ is the asymptotic value of the dilaton, we find the explicit form of the loop corrections to the tree-level metric and moduli of a general dyonic solution.

To simplify solution of the loop-corrected equations, we consider a particular tree-level dyonic black-hole solution with the constant metric $G_{m n}$ of the two-torus $T^{2}$ and vanishing antisymmetric tensor $B_{12}$. With this choice, the standard tree-level moduli $T$ and $U$ are independent of coordinates, but dilaton and space-time metric are coordinates-dependent. This technical simplification makes possible to avoid too cumbersome expressions for solutions to the system of the Maxwell and Killing spinor equations and to obtain explicit expressions for the loop corrections to the metric and moduli.

Loop corrections to the metric and dilaton are expressed via the unambiguous real part of the loop correction to the prepotential $\operatorname{Re} h(T, U, \bar{T}, \bar{U})$. The loop-corrected dyonic metric is

$$
g_{00}=-g^{i i}=\frac{r^{2}}{(r+P)(r+Q)}\left(1-\epsilon \frac{P H}{r+Q}\right),
$$

where $H=R e h / 2 T U=\operatorname{Re} h / 2 G_{11}, P=\left(8 P^{0} P^{1}\right)^{1 / 2}, Q=\left(8 Q_{2} Q_{3}\right)^{1 / 2}$ and $P^{0}, P^{1}$ and $Q_{2}, Q_{3}$ are electric and magnetic charges of the dyonic black hole, $T$ and $U$ are the standard moduli calculated with the tree-level dyonic solution. Loop corrections to the two-torus metric are

$$
\delta G_{11}=\epsilon C_{1} \frac{r}{r+P}, \quad \delta G_{22}=\epsilon\left(C_{2} \frac{r}{r+Q}-\left(L_{2}-L_{3}\right) \frac{P}{r+Q}\right)
$$

and are finite for all $r$. Here $C_{i}$ are arbitrary constants, $L_{2}=\partial_{T} R e h / U$ and $L_{3}=\partial_{U} R e h / T$.

In the case of magnetic black hole, the string-tree-level dilaton increases at small distances from the origin, and the loop corrections blow up at the origin. In electric case and for a general dyonic solution with two electric and two magnetic charges, dilaton is finite in the whole spacetime, and corrections to the tree-level metric and moduli are coordinate-dependent, but finite.

Although the tree-level dyonic solution is static, with vanishing axions (imaginary parts of the moduli) and four charges, in the loop-corrected solution, generically, can appear new fields of the first order in string coupling, in particular, axions and new vector fields. At the same time, generically, the loop-corrected solution becomes stationary, with the stationarity coefficients of the first order in string coupling. However, for a suitable choice of arbitrary constants there exists a stationary loop-corrected solution with four charges, but with non-vanishing axions.

In Sect. 2 we review direct path-integral calculation of string-loop corrections to various terms of the universal sector of the low-energy effective action performing integration over the world sheets of torus topology.

In Sect. 3 we discuss formulation of the theory based on $N=2$ local supersymmetry. In this approach, dynamics of the theory is defined in terms of the prepotential of the theory. The 
prepotential of $N=2$ locally supersymmetric theory receives string-loop correction only from the world sheets of torus topology $[12,16,17,18]$. Using the loop-corrected prepotential, we calculate the gauge couplings in the first order in string coupling and the ambiguities in the gauge couplings due to the ambiguity in the loop correction to the prepotential. In perturbative approach, corrections to the gauge couplings and to the Kähler potential for the moduli, which are of the first order in string coupling, are calculated by substituting the tree-level moduli.

We find the form of the loop-corrected symplectic transformation connecting the holomorphic section with the prepotential and that associated with the heterotic string compactification.

In Sect. 4 we derive the Killing spinor equations for the gravitino and the fermionic superpartners of the moduli and present them in different forms convenient for subsequent solution.

In Sect.5, solving the combined system of the Killing spinor and Maxwell equations we obtain the tree-level dyonic black hole which in the following we use as the in-put in calculations of the loop corrections.

In Sect.6, in the first order in string coupling constant, we obtain the loop-corrected solution for the field strengths, metric and moduli. Although the gauge couplings contain the ambiguities, in the field strengths they cancel.

In Sect.7 we consider the loop-corrected expressions for the BPS and ADM masses of the black hole. As expected in supersymmetric theories, for solutions with partially broken supersymmetry the ADM mass obtained from the asymptotic form of the metric is equal to the BPS mass.

In Sect. 8 we discuss the equations for the axions which are the imaginary parts of the gaugini Killing spinor equations. The metric components responsible for non-stationarity of solution are expressed as functions of the axions. It is shown that there is a solution for the axions for which the metric remains static.

\section{String-loop corrected $N=2$ supersymmetric effective action}

The bosonic part of the universal sector of the $6 D$ effective action of the heterotic string theory compactified to six dimensions with $N=1$ supersymmetry on the manifold $K 3$ or on a suitable orbifold is

$$
I_{6}=\frac{1}{2 \kappa_{6}{ }^{2}} \int d^{6} x \sqrt{-G^{(6)}} e^{-\Phi^{\prime}}\left[R^{(6)}+\left(\partial \Phi^{\prime}\right)^{2}-\frac{H^{2}}{12}\right]+\ldots
$$

Further compactification on a two-torus yields the $4 D$ theory with $N=2$ supersymmetry. The standard decomposition of the $6 D$ metric is

$$
G^{(6)}=\left(\begin{array}{cc}
G_{\mu \nu}+A_{\mu}^{m} A_{\nu}^{n} G_{m n} & A_{\mu}^{m} G_{m n} \\
A_{\nu}^{n} G_{m n} & G_{m n}
\end{array}\right) .
$$

Dimensional reduction of the action (1) yields [19]

$$
I_{4}=\frac{1}{2 \kappa_{4}^{2}} \int d^{4} x \sqrt{-G^{(4)}} e^{-\phi^{\prime}}\left[R\left(G^{\prime}\right)+\left(\partial \phi^{\prime}\right)^{2}-\frac{\left(H^{\prime}\right)^{2}}{12}+\frac{1}{4} \mathcal{F}(L M L) \mathcal{F}+\frac{1}{8} \operatorname{Tr}(\partial M L \partial M L)\right],
$$


where $\mu, \nu=0, \ldots, 3$ and $m, n=1,2$. The second pair of the vector fields are the components $B_{m \mu}$ of the antisymmetric field $B^{(6)}$.

Here

$$
M=\left(\begin{array}{cc}
G^{-1} & G^{-1} B \\
-B G^{-1} & G
\end{array}\right), \quad L=\left(\begin{array}{cc}
0 & I_{2} \\
I_{2} & 0
\end{array}\right)
$$

and

$$
\begin{aligned}
G^{\prime} & \equiv G_{\mu \nu}^{\prime}=G_{\mu \nu}+A_{\mu}^{m} A_{\nu}^{n} G_{m n}, \quad G \equiv G_{m n} \\
H^{\prime} & \equiv H_{\mu \nu \lambda}^{\prime}=H_{\mu \nu \lambda}-\left(A^{(1) n} H_{n \nu \lambda}-A^{(1) m} A^{(1) n} H_{m n \lambda}+\text { cycl.perms. }\right) .
\end{aligned}
$$

A direct way to calculate corrections to the string-tree-level effective action from integration over the string world sheets of torus topology is to perform the path integrals for correlators yielding the relevant structures in the effective action. For heterotic string theory, The part of the world-sheet action of the heterotic string theory which depends on background fields from the universal sector is

$$
I_{1,0}=\int d^{2} z d \vartheta\left(G_{M N}+B_{M N}\right)(\hat{X}) D \hat{X}^{M} \bar{\partial} \hat{X}^{N}
$$

Here $I_{1,0}$ is the action with the $(1,0)$ supersymmetry in the left supersymmetric sector, $\hat{X}^{M}=$ $X^{M}+\vartheta \psi^{M}, M=0, \ldots, 9, D=\partial_{\vartheta}+\vartheta \partial_{z}$.

Performing integration over $\vartheta$ one arrives at the action

$$
\begin{aligned}
I_{1,0}= & \int d^{2} z\left(G_{\mu \nu}+B_{\mu \nu}\right)\left(\partial X^{\mu} \bar{\partial} X^{\nu}-\psi^{\mu} \bar{\partial} \psi^{\nu}\right)+\left(G_{\mu n}+B_{\mu n}\right)\left(\partial X^{\mu} \bar{\partial} X^{n}-\psi^{\mu} \bar{\partial} \psi^{n}\right) \\
+\left(G_{m \nu}+\right. & \left.B_{m \nu}\right)\left(\partial X^{m} \bar{\partial} X^{\nu}-\psi^{m} \bar{\partial} \psi^{\nu}\right)+\left(G_{m n}+B_{m n}\right)\left(\partial X^{m} \bar{\partial} X^{n}-\psi^{m} \bar{\partial} \psi^{n}\right) \\
+\left(G_{\mu \nu}+\right. & \left.B_{\mu \nu}\right)_{, \rho} \psi^{\rho} \psi^{\mu} \bar{\partial} X^{\nu}+\left(G_{\mu n}+B_{\mu n}\right)_{, \rho} \psi^{\rho} \psi^{\mu} \bar{\partial} X^{n}+\left(G_{m \nu}+B_{m \nu}\right)_{, \rho} \psi^{\rho} \psi^{m} \bar{\partial} X^{\nu} \\
& +\left(G_{m n, \rho}+B_{m n, \rho}\right) \psi^{\rho} \psi^{m} \bar{\partial} X^{n} .
\end{aligned}
$$

To be concrete, we have in view heterotic string theory compactified to four dimensions on the orbifold $T^{4} / Z_{2} \times T^{2}$. The partition function has the form of the sum of terms, where each term is the product of contributions from integration over bosonic and fermionic variables.

Correlator of free bosons on the world sheet of torus topology with the Teichmuller parameter $\tau$ is given by [4]

$$
<X(z . \bar{z}) X(0)>=-\log \left|\vartheta_{1}(z)\right|^{2}-\frac{\pi}{2 \tau_{2}}(\operatorname{Im} z)^{2}
$$

Correlator of fermions with even spin structures $a, b \neq 1,1$ is ${ }^{2}$

$$
S\left[\begin{array}{l}
a \\
b
\end{array}\right](z)=<\psi(z) \psi(0)>=\frac{\vartheta\left[\begin{array}{l}
a \\
b
\end{array}\right](z) \vartheta_{1}^{\prime}(0)}{\vartheta\left[\begin{array}{l}
a \\
b
\end{array}\right](0) \vartheta_{1}(z)}
$$

\footnotetext{
${ }^{2}$ We consider only fermions with even spin structures which yield non-vanishing contributions to the correlators.
} 
where $\vartheta\left[\begin{array}{l}1 \\ 1\end{array}\right]=\vartheta_{1}$.

Contribution from the integration over the left-moving fermions (in the light-cone gauge) yields the blocks of the form (see, for example, [9])

$$
\sum_{a, b=0}^{1}(-)^{a+b+a b} \frac{\vartheta^{2}\left[\begin{array}{l}
a \\
b
\end{array}\right](0) \vartheta\left[\begin{array}{l}
a+h \\
b+g
\end{array}\right](0) \vartheta\left[\begin{array}{l}
a-h \\
b-g
\end{array}\right](0)}{\eta^{4}}
$$

In untwisted case, eight left-moving fermions have the same spin structures, and the resulting $4 D$ theory has $N=4$ supersymmetry. Shifts $h, g$, taking values 0,1 , appear from the orbifold construction and reduce supersymmetry to $N=2$.

Performing in (9) summation over spin structures and using the Jacobi identities, one has

$$
\frac{1}{2} \sum_{a, b=0}^{1}(-)^{a+b+a b} \frac{\vartheta^{2}\left[\begin{array}{l}
a \\
b
\end{array}\right](0) \vartheta\left[\begin{array}{l}
a+h \\
b+g
\end{array}\right](0) \vartheta\left[\begin{array}{l}
a-h \\
b-g
\end{array}\right](0)}{\eta^{4}}=-\vartheta^{2}\left[\begin{array}{l}
1 \\
1
\end{array}\right] \vartheta\left[\begin{array}{l}
1-h \\
1-g
\end{array}\right] \vartheta\left[\begin{array}{l}
1+h \\
1+g
\end{array}\right](0)=0
$$

because $\vartheta_{1}(0)=0$.

Let us show that the Einstein term receives no corrections from the world sheets of torus topology. The graviton vertex function is $[1,4,9]$

$$
V_{G}=\int d^{2} z G_{\mu \nu}(X)\left(\partial X^{\mu}+i(p \psi) \psi^{\mu}\right) \bar{\partial} X^{\nu} e^{i p X}
$$

Let us calculate the two-point correlator of the vertex functions (11) and take the $O\left(p^{2}\right)$ piece 3. Due to (10), purely bosonic part of the correlator $\left\langle V_{G} V_{G}\right\rangle$ vanishes. The four-fermion part is proportional to the expression

$$
\frac{1}{2} \sum_{a, b \neq 1,1}^{1}(-)^{a+b+a b} \frac{\vartheta^{2}\left[\begin{array}{l}
a \\
b
\end{array}\right](0) \vartheta\left[\begin{array}{l}
a+h \\
b+g
\end{array}\right](0) \vartheta\left[\begin{array}{l}
a-h \\
b-g
\end{array}\right](0)}{\eta^{4}} S^{2}\left[\begin{array}{l}
a \\
b
\end{array}\right](z)=4 \pi^{2} \eta^{2} \vartheta\left[\begin{array}{l}
1-h \\
1-g
\end{array}\right] \vartheta\left[\begin{array}{l}
1+h \\
1+g
\end{array}\right](0),
$$

where again we used the Jacobi identity and the relation $\vartheta_{1}^{\prime}=-2 \pi \eta^{3}$. Expression (12) is independent of $z$. The integral over the $z$-dependent part of the correlator is

$$
\int d^{2} z<\bar{\partial} X(z) \bar{\partial} X(0)>=-\int d^{2} z\left(\bar{\partial}^{2} \log \bar{\vartheta}_{1}+\frac{\pi}{\tau_{2}}\right)=0 .
$$

Thus, the $O\left(p^{2}\right)$ piece of the correlator $\left\langle V_{G} V_{G}>\right.$ vanishes, and there is no correction to the Einstein term. The same is true for the vertex $V_{B}$ with $G_{\mu \nu}$ substituted by $B_{\mu \nu}$.

Let us consider the correlators of the gauge vertices $[4,7,9]$

$$
V_{A}=\int d^{2} z G_{p q}(X)\left(A_{\mu}^{p}(X) \partial X^{\mu}+\frac{1}{2} F_{\mu \nu}^{p} \psi^{\mu} \psi^{\nu}\right) \bar{\partial} X^{q} e^{i p X}
$$

(the same with $A_{\mu}^{p}$ substituted by $B_{p \mu}$ ), where $p, q=1,2$ label the directions of the untwisted torus $T^{2}$. Non-vanishing contribution of the left-moving fields is produced by the fermionic

\footnotetext{
${ }^{3}$ It is understood that the wave function factors making the amplitude vanishing on-shell are removed.
} 
terms in the vertices $V_{A}$. In contrast to the graviton vertex, in the present case, the rightmoving bosons $X^{p}$ carry not $4 D$, but an internal $T^{2}$ index. The fields $X^{p}$ can be split into the classical

$$
\tilde{X}^{p}(m, n)=\pi R\left[\left(m^{p}-n^{p} \bar{\tau}\right) \frac{z}{i \tau_{2}}-\left(m^{p}-n^{p} \tau\right) \frac{\bar{z}}{i \tau_{2}}\right]
$$

and a quantum parts $Y^{p}: X^{p}=\tilde{X}^{p}+Y^{p}$. Here it is assumed that the classical fields $\tilde{X}^{p}$ take values on the circles of the radius $R: \tilde{X}^{p} \sim \tilde{X}^{p}+2 \pi R k$. Contribution of the right-moving bosons is

$$
\sum_{(m, n)} e^{-S(m, n)-I(Y)}\left[\bar{\partial} \tilde{X}^{p} \bar{\partial} \tilde{X}^{q}+<\bar{\partial} Y^{p} \bar{\partial} Y^{q}>\right]
$$

where the classical action is

$$
S(m, n)=\frac{\pi R^{2} G_{p q}}{\tau_{2}}\left(m^{p}-n^{p} \bar{\tau}\right)\left(m^{q}-n^{q} \tau\right)
$$

and $I(Y)=\int d^{2} z G_{p q} \partial Y^{p} \bar{\partial} Y^{q}$. As in the case of the graviton vertex, the integral over bosonic correlator $<\bar{\partial} Y^{p} \bar{\partial} Y^{q}>$ vanishes. The classical part is non-zero and produces a one-loop correction to the tree-level term $G_{p q} F_{\mu \nu}^{p} F^{q \mu \nu}$.

In the following, instead of performing path-integral calculations of the string-loop corrections to various terms of the action ${ }^{4}$, we shall make use of the $N=2$ formulation of the theory, in which different loop corrections are expressed via the loop-corrected prepotential.

\section{$3 \quad \mathrm{~N}=2$ formulation of the effective field theory}

\subsection{Heterotic string effective action and prepotential of the STU model}

The dilaton $\phi^{\prime}$ in $(3)$ can be split into the sum of the constant part $\phi_{\infty}$ and a term vanishing at spatial infinity $\phi^{\prime}=\phi_{\infty}+\phi$. In string perturbation theory, higher order contributions enter with the factor $e^{\frac{1}{2} \chi \phi}$, where $\chi$ is the Euler characteristic of the string world sheet [24]. The exponent $e^{\phi_{\infty}} \equiv \epsilon$ can be considered as a string-loop expansion parameter.

Written in the Einstein frame, where $g_{\mu \nu}=e^{-\phi} G_{\mu \nu}^{\prime}$, the action (3) is [19]

$$
I_{4}=\int d^{4} x \sqrt{-g}\left[R-\frac{1}{2}(\partial \phi)^{2}-\frac{e^{-\phi}}{4} \mathcal{F}(L M L) \mathcal{F}+\frac{a_{1}}{4 \sqrt{-g}} \mathcal{F} L^{*} \mathcal{F}+\frac{1}{8} \operatorname{Tr}(\partial M L \partial M L)+\ldots\right] .
$$

The $4 D$ dilaton $\phi$ and axion $a_{1}$ are defined as

$$
\phi=\Phi-\frac{1}{2} \ln \operatorname{det}\left(G_{m n}\right), \quad \partial_{\rho} a_{1}=-H^{\prime \mu \nu \lambda} e^{-2 \phi} \sqrt{-g} e_{\mu \nu \lambda \rho}
$$

\footnotetext{
${ }^{4}$ The main difficulty in these calculations is performing the sum over classical configurations in (16) (cf. with calculations in [7], where, in distinction to the present case, the pre-exponent factor was $\left.\partial \tilde{X}^{p} \bar{\partial} \tilde{X}^{q}\right)$.
} 
The bosonic part of the $4 D$ action written in the standard form of $N=2$ supersymmetric theory is (for example, $[20,21,22,23]$ and references therein)

$$
I_{4}^{N=2}=\int d^{4} x \sqrt{-g}\left[\frac{1}{2} R+\left(\bar{N}_{I J} \mathcal{F}^{-I} \mathcal{F}^{-J}-N_{I J} \mathcal{F}^{+I} \mathcal{F}^{+J}\right)+k_{i \bar{j}} \partial_{\mu} y_{i} \partial^{\mu} \bar{y}_{j}+\ldots\right],
$$

where

$$
\mathcal{F}_{\mu \nu}^{ \pm}=\frac{1}{2}\left(\mathcal{F}_{\mu \nu} \pm i \sqrt{-g} \mathcal{F}_{\mu \nu}\right)=\frac{1}{2}\left(\mathcal{F}_{\mu \nu} \pm \frac{i}{2} \epsilon_{\mu \nu \rho \lambda} \mathcal{F}^{\rho \lambda}\right)
$$

Here ${ }^{*} \mathcal{F}_{\mu \nu}=\frac{1}{2} e_{\mu \nu \rho \lambda} \mathcal{F}^{\rho \lambda}$, where $e_{\mu \nu \rho \lambda}$ is the flat antisymmetric tensor, $e_{0123}=-1$. The gauge coupling constants $N_{I J}$ are defined below. The Kähler metric is

$$
k_{i \bar{j}}=\frac{\partial^{2} K^{(0)}}{\partial y_{i} \partial \bar{y}_{j}}
$$

where $y_{i}$ are the tree-level moduli, and the tree-level Kähler potential $K^{(0)}$ is

$$
K^{(0)}=-\log \left(y_{1}+\bar{y}_{1}\right)\left(y_{2}+\bar{y}_{2}\right)\left(y_{3}+\bar{y}_{3}\right) .
$$

In the case of the $N=2$ supersymmetric compactification of superstring theory dynamics of the theory is encoded in the prepotential which tree-level part is

$$
F=-\frac{X^{1} X^{2} X^{3}}{X^{0}}+\ldots
$$

where

$$
\begin{gathered}
\frac{X^{1}}{X^{0}}=i y_{1}=i\left(e^{-\phi}+i a_{1}\right), \\
\frac{X^{2}}{X^{0}}=i y_{2}=i\left(e^{\gamma+\sigma}+i a_{2}\right), \\
\frac{X^{3}}{X^{0}}=i y_{3}=i\left(e^{\gamma-\sigma}+i a_{3}\right)
\end{gathered}
$$

and dots stand for contributions from other moduli. Here and below $I, J=0, \ldots, 3$ and $i, j=1,2,3$.

The moduli $y_{i}$ are equal to conventional moduli $S, T, U$ :

$$
\left(y_{1}, y_{2}, y_{3}\right)=\left(S=e^{-\phi}+i a_{1}, T=\sqrt{G}+i B_{12}, U=\frac{\left(\sqrt{G}+i G_{12}\right)}{G_{22}}\right) .
$$

The axion $a_{1}$ is defined in (18), $a_{2}=B_{12}$, and $\gamma, \sigma$ and $a_{3}$ are read off by comparing definitions (21) with parametrization of the metric components of the two-torus

$$
G_{m n}=e^{2 \sigma}\left(\begin{array}{cc}
e^{2 \gamma-2 \sigma}+a_{3}^{2} & -a_{3} \\
-a_{3} & 1
\end{array}\right)
$$


The gauge part of the action (3) with $B_{12}=0$ and diagonal metric $G_{m n}$ is

$$
-\frac{1}{4} G_{11}\left(\mathcal{F}^{(1) 1}\right)^{2}-\frac{1}{4} G_{22}\left(\mathcal{F}^{(1) 2}\right)^{2}-\frac{1}{4} G^{11}\left(\mathcal{F}_{1}^{(2)}\right)^{2}-\frac{1}{4} G^{22}\left(\mathcal{F}_{2}^{(2)}\right)^{2},
$$

where

$$
\mathcal{F}_{\mu \nu}^{(1) m}=\partial_{\mu} A_{\nu}^{m}-\partial_{n} A_{\mu}^{m}, \quad \mathcal{F}_{m \mu \nu}^{(2)}=\partial_{\mu} B_{m \nu}-\partial_{n} B_{m \mu}
$$

It is convenient to relabel the vector fields in correspondence with the moduli with which they form the superfields

$$
A_{\mu}^{1}=\sqrt{8} \mathcal{A}_{\mu}^{0}, \quad B_{1 \mu}=\sqrt{8} \mathcal{A}_{\mu}^{1}, \quad A_{\mu}^{2}=\sqrt{8} \mathcal{A}_{\mu}^{2} \quad B_{2 \mu}=\sqrt{8} \mathcal{A}_{\mu}^{3} .
$$

The factor $\sqrt{8}$ appears because of different normalizations of the gauge field in the actions (17) and (19).

\subsection{Loop-corrected gauge couplings}

In the case of the $N=2$ supersymmetric compactification of superstring theory, the prepotential receives only only one-string-loop correction $[12,13,16,23]$

$$
F=-\frac{X^{1} X^{2} X^{3}}{X^{0}}-i \epsilon X^{0} \epsilon h\left(-i \frac{X^{2}}{X^{0}},-i \frac{X^{3}}{X^{0}}\right)+\ldots
$$

which is independent of the modulus $X^{1}$. In the following, we do not rely on the explicit form of the loop correction calculated in $[8,16,17]$.

The Kähler potential becomes

$$
K=-\ln \left[\left(y_{1}+\bar{y}_{1}+\epsilon V\right)\left(y_{2}+\bar{y}_{2}\right)\left(y_{3}+\bar{y}_{3}\right)\right],
$$

where the Green-Schwarz function $[8,12,16,18]$

$$
V\left(y_{2}, \bar{y}_{2}, y_{3}, \bar{y}_{3}\right)=\frac{\operatorname{Re} h-\operatorname{Re} y_{2} \operatorname{Re} \partial_{y_{2}} h-\operatorname{Re} y_{3} \operatorname{Re} \partial_{y_{3}} h}{\operatorname{Re} y_{2} \operatorname{Re} y_{3}}
$$

is of the first order in string coupling. Beyond the tree level, the modulus $S=y_{1}$ mixes with other moduli, and its one-loop form is [18]

$$
S=e^{-\phi}-\epsilon \frac{V}{2}+i a_{1}
$$

In sections which admit the prepotential, the gauge coupling constants in the action (19) are calculated using the formula [10]

$$
N_{I J}=\bar{F}_{I J}+2 i \frac{\left(I m F_{I K} X^{K}\right)\left(I m F_{J L} X^{L}\right)}{\left(X^{I} I m F_{I J} X^{J}\right)},
$$

where $F_{I}=\partial_{X^{I}} F, F_{I J}=\partial_{X^{I} X^{J}}^{2} F$, etc. 
Having in view application of general formulas to dyonic black hole solutions in which case the tree-level moduli $y_{i}$ are real, in the first order in string coupling constant, we obtain the gauge couplings $N_{I J}$ as

$$
\begin{aligned}
& N_{00}=i y^{3}\left(-1+\epsilon \frac{n}{4 y^{3}}\right), \quad N_{01}=-\epsilon \frac{n+2 v}{4 y_{1}}-i \epsilon a_{1} \frac{y_{2} y_{3}}{y_{1}}, \\
& N_{02}=-\epsilon \frac{n+2 v-2 y_{2} h y+4 y_{2} h_{2}}{4 y_{2}}-i \epsilon a_{2} \frac{y_{1} y_{3}}{y_{2}}, \\
& N_{03}=-\epsilon \frac{n+2 v-2 y_{3} h y+4 y_{3} h_{3}}{4 y_{3}}-i \epsilon a_{3} \frac{y_{1} y_{2}}{y_{3}}, \\
& N_{11}=-i \frac{y^{3}}{y_{1}^{2}}\left(1+\epsilon \frac{n}{4 y^{3}}\right), \quad N_{12}=i \epsilon y_{3} \frac{2 y_{2} h y-n}{4 y^{3}}+\epsilon a_{3}, \quad N_{13}=i \epsilon y_{2} \frac{2 y_{3} h y-n}{4 y^{3}}+\epsilon a_{2}, \\
& N_{22}=-i \frac{y^{3}}{y_{2}^{2}}\left(1-\epsilon\left(\frac{y_{2} h_{23} y_{3}}{y^{3}}+\frac{n}{4 y^{3}}\right)\right), \quad N_{33}=-i \frac{y^{3}}{y_{3}^{2}}\left(1-\epsilon\left(\frac{y_{2} h_{23} y_{3}}{y^{3}}+\frac{n}{4 y^{3}}\right)\right), \\
& N_{23}=i \epsilon y_{1} \frac{2 y h y-4 y_{2} h_{23} y_{3}-n}{4 y^{3}}+\epsilon a_{1} .
\end{aligned}
$$

Here we kept notations $y_{i}$ for the real parts of the moduli, the imaginary parts (axions) $a_{i}$ are of the first order in the string coupling. The following notations are introduced

$$
y^{3}=y_{1} y_{2} y_{3}, \quad h y=h_{a} y_{a}=h_{2} y_{2}+h_{3} y_{3}, \quad h_{a}=\partial_{y_{a}} h, \quad h_{a b}=\partial_{y_{a}} \partial_{y_{b}} h
$$

and

$$
v=h-y_{a} h_{a}, \quad n=h-h_{a} y_{a}+y_{a} h_{a b} y_{b}, \quad y_{2} h y=y_{2} h_{2 a} y_{b} .
$$

The terms of the first order in string coupling $\epsilon$ are linear functions of the loop correction to the prepotential $h$ and are calculated by substituting the tree-level moduli obtained from the input tree-level dyonic black hole solution [31, 32] and also [22, 23, 28, 35] and refs. therein. The imaginary parts $a_{i}$ of the moduli $y_{i}$ which are absent in the classical dyonic solution can appear in loop-corrected solutions of field equations in the next order in string coupling constant.

Deriving the above expressions we assumed that the function $h\left(y_{2}, y_{3}\right)$ is real. Explicit calculation [17] (see Sect.6.4) shows that the function $h$ contains an unambiguous imaginary polynomial part which we combine with the imaginary ambiguities discussed below.

The loop correction to the prepotential is defined up to an arbitrary quadratic polynomial $P\left(y_{2}, y_{3}\right)$ in variables $y_{2}, y_{3}$ and $y_{2} y_{3}$ with imaginary coefficients $[16,17,12]$. Application of the formula (29) shows that for real $y_{2}, y_{3}$ and real function $h\left(y_{2}, y_{3}\right)$ the ambiguities of the couplings $N_{I J}$ contain the extra factor $i$ with respect to the unambiguous part.

Introducing

$b=4\left(P_{a} y_{a}-y_{a} P_{a b} y_{b}\right), \quad b_{2}=4\left(P_{2}-y_{a} P_{a 2}\right), \quad b_{3}=4\left(P_{3}-y_{a} P_{a 3}\right), \quad P_{00}=2 P-2 y_{a} P_{a}+y_{a} P_{a b} y_{b}$, where we used the same notations as above in (31), we obtain the ambiguity of the couplings 
as

$$
\delta N_{I J}=\left(\begin{array}{cccc}
P_{00}+3 b & -\frac{i b}{y_{1}} & \frac{i\left(2 b_{2} y_{2}-b\right)}{y_{2}} & \frac{i\left(2 b_{3} y_{3}-b\right)}{y_{3}} \\
-\frac{i b}{y_{1}} & \frac{b}{y_{1}^{2}} & \frac{\left(b-2 b_{2} y_{2}\right)}{y_{1} y_{2}} & \frac{\left(b-2 b_{3} y_{3}\right)}{y_{1} y_{3}} \\
\frac{i\left(2 b_{2} y_{2}-b\right)}{y_{2}} & \frac{\left(b-2 b_{2} y_{2}\right)}{y_{1} y_{2}} & -P_{22}+\frac{\left(b-4 b_{2} y_{2}\right)}{y_{2}^{2}} & -P_{23}-\frac{b}{y_{2} y_{3}} \\
\frac{i\left(2 b_{3} y_{3}-b\right)}{y_{3}} & \frac{\left(b-2 b_{3} y_{3}\right)}{y_{1} y_{3}} & -P_{23}-\frac{b}{y_{2} y_{3}} & -P_{33}+\frac{\left(b-4 b_{3} y_{3}\right)}{y_{3}^{2}}
\end{array}\right) .
$$

Note that the gauge couplings of the vector fields in the universal sector residing from the moduli supermultiplets have different structure from the Wilsonian couplings of the gauge fields in the matter sector originating from the terms $\left[f_{a b} W^{a} W^{b}\right]_{F}$ in the locally supersymmetric action $[18]$.

\subsection{Symplectic transformations}

The field equations and the Bianchi identities for the gauge field strengths are

$$
\begin{aligned}
\partial_{\mu}\left(\sqrt{-g} \operatorname{Im} G_{I}^{-\mu \nu}\right) & =0 \\
\partial_{\mu}\left(\sqrt{-g} I m \mathcal{F}^{-J \mu \nu}\right) & =0
\end{aligned}
$$

where $G_{I}^{-\mu \nu}=\bar{N}_{I J} \mathcal{F}^{-J \mu \nu}$. Eqs.(34) and are invariant under the symplectic $S p(8, \mathbf{Z})$ transformations

$$
O=\left(\begin{array}{cc}
A & B \\
C & D
\end{array}\right)
$$

where

$$
A^{T} C-C^{T} A=0, \quad B^{T} D-D^{T} B=0, \quad A^{T} D-C^{T} B=1 .
$$

Under symplectic transformations the couplings $N_{I J}$ are transformed as

$$
\hat{N}=(C+D N)(A+B N)^{-1} .
$$

In sections which do not admit a prepotential (including that which naturally appears in $4 D$ compactification of the heterotic string), the gauge couplings are calculated by making a symplectic transformation of the couplings (30) calculated in the section with the prepotential. Specifically, at the tree level, the section connected with compactification of the heterotic string from $6 D$ to $4 D$ is obtained from that with the prepotential (26) by symplectic transformation $[12]$

$$
A=\operatorname{diag}(1,0,1,1), B=\operatorname{diag}(0,1,0,0), C=\operatorname{diag}(0,-1,0,0), D=\operatorname{diag}(1,0,1,1) .
$$

At the one-loop level, we look for a symplectic transformation connecting heterotic section with that with the prepotential in the form

$$
\begin{gathered}
A=\operatorname{diag}(1,0,1,1)+\epsilon\left(a_{i j}\right), B=\operatorname{diag}(0,1,0,0)+\epsilon\left(b_{i j}\right), \\
C=\operatorname{diag}(0,-1,0,0)+\epsilon\left(c_{i j}\right), D=\operatorname{diag}(1,0,1,1)+\epsilon\left(d_{i j}\right) .
\end{gathered}
$$


The matrices $a, b, c$ and $d$ are constrained by relations (36).

In the case of tree-level solutions with vanishing axionic parts, the tree-level gauge couplings $N_{00}=-i y_{1} y_{2} y_{3}, \quad N_{22}=-i \frac{y_{1} y_{3}}{y_{2}}, \quad N_{33}=-i \frac{y_{1} y_{2}}{y_{3}}$ are proportional to $e^{-\phi}$, whereas the expression $N_{11}=-i \frac{y_{2} y_{3}}{y_{1}}$ contains the factor $e^{\phi}$. In the heterotic section, the tree-level gauge couplings are proportional to $y_{1}=e^{-\phi}$. Loop corrections to the tree-level couplings, as well as non-diagonal couplings absent at the tree level, appear with the extra factor $\epsilon e^{\phi}$.

We require that the loop-corrected symplectic transformations (39) produce the same structure of loop corrections to the gauge couplings in the heterotic section as that which appear in path-integral calculation of loop corrections, i.e. loop corrections have the extra factor $\epsilon e^{\phi}$ as compared to the tree-level expressions.

Because we perform calculations with accuracy up to the terms of the first order in string coupling, corrections to to the gauge couplings due to the one-loop term in the prepotential and to the loop corrections to the tree-level symplectic transformations can be treated independently. From the relations (36) we find that admissible non-zero entries are $c_{i j}$ with $c_{1 i}=c_{i 1}=0$ and $d_{11}$.

Calculating the matrix of the loop-corrected gauge couplings in the heterotic section and requiring that it is of the form discussed above, we find

$$
\hat{N}_{I J}=\left(\begin{array}{cccc}
N_{00}+\epsilon c_{00}-\frac{N_{01}^{2}}{N_{11}} & \frac{N_{01}}{N_{11}} & N_{02}+\epsilon c_{02}-\frac{N_{01} N_{12}}{N_{11}} & N_{03}+\epsilon c_{03}-\frac{N_{01} N_{13}}{N_{11}} \\
\frac{N_{10}}{N_{11}} & -\frac{1}{N_{11}}+\epsilon d_{11} & \frac{N_{12}}{N_{11}} & \frac{N_{13}}{N_{11}} \\
N_{20}+\epsilon c_{20}-\frac{N_{21} N_{10}}{N_{11}} & \frac{N_{21}}{N_{11}} & N_{22}+\epsilon c_{22}-\frac{N_{21}^{2}}{N_{11}} & N_{23}+\epsilon c_{23}-\frac{N_{21} N_{13}}{N_{11}} \\
N_{30}+\epsilon c_{30}-\frac{N_{31} N_{10}}{N_{11}} & \frac{N_{31}}{N_{11}} & N_{32}+\epsilon c_{23}-\frac{N_{31} N_{12}}{N_{11}} & N_{33}+\epsilon c_{33}-\frac{N_{31}^{2}}{N_{11}}
\end{array}\right) .
$$

where $c_{i j}$ and $d_{11}$ are real constants which shift the topological term in the action and do not affect the equations of motion.

Since at the tree level there are only diagonal couplings, the non-diagonal terms are of next order in $\epsilon$. The terms of the form $\frac{N_{1 I} N_{1 J}}{N_{11}}$ are also of the next order in the string coupling.

In the basis associated with the heterotic string compactification, the part of the matrix $\hat{N}_{I J}$ which contains the imaginary parts of the moduli $a_{i}$ and ambiguities is

$$
\left(\begin{array}{cccc}
P_{00}+3 b & a_{1}+\frac{b}{y_{2} y_{3}} & -\frac{i a_{2} y_{1} y_{3}}{y_{2}}-\frac{i\left(b-2 b_{2} y_{2}\right)}{y_{2}} & -\frac{i a_{3} y_{1} y_{2}}{y_{3}}-\frac{i\left(b-2 b_{3} y_{3}\right)}{y_{3}} \\
a_{1}+\frac{b}{y_{2} y_{3}} & -\frac{b}{\left(y_{2} y_{3}\right)^{2}} & \frac{i a_{3} y_{1}}{y_{2} y_{3}}+\frac{i\left(b-2 b_{2} y_{2}\right)}{y_{2}^{2} y_{3}} & \frac{i a_{2} y_{1}}{y_{2} y_{3}}-\frac{i\left(b-2 b_{3} y_{3}\right)}{y_{3}^{2} y_{2}} \\
-\frac{i a_{2} y_{1} y_{3}}{y_{2}}-\frac{i\left(b-2 b_{2} y_{2}\right)}{y_{2}} & \frac{i a_{3} y_{1}}{y_{2} y_{3}}+\frac{i\left(b-2 b_{2} y_{2}\right)}{y_{2}^{2} y_{3}} & -P_{22}-\frac{\left(4 b_{2}-b\right)}{y_{2}^{2}} & a_{1}-P_{23}-\frac{b}{y_{2} y_{3}} \\
-\frac{i a_{3} y_{1} y_{2}}{y_{3}}-\frac{i\left(b-2 b_{3} y_{3}\right)}{y_{3}} & \frac{i a_{2} y_{1}}{y_{2} y_{3}}+\frac{i\left(b-2 b_{2} y_{2}\right)}{y_{3}^{2} y_{2}} & a_{1}-P_{23}-\frac{b}{y_{2} y_{3}} & P_{33}-\frac{\left(4 b_{3} y_{3}-b\right)}{y_{3}^{2}}
\end{array}\right)
$$

From the symplectic transformation of the field strengths

$$
\left(\begin{array}{c}
\hat{\mathcal{F}}^{-} \\
\hat{G}^{-}
\end{array}\right)=O\left(\begin{array}{c}
\mathcal{F}^{-} \\
G^{-}
\end{array}\right)
$$


we obtain the relation between the field strengths in the section with the prepotential and those which appear in the heterotic string effective action in the form

$$
\begin{gathered}
\hat{\mathcal{F}}^{-0}=\mathcal{F}^{-0}, \quad \hat{\mathcal{F}}^{-2}=\mathcal{F}^{-2}, \quad \hat{\mathcal{F}}^{-3}=\mathcal{F}^{-3} \\
\hat{G}_{0}^{-}=G_{0}^{-}+\epsilon\left(c_{00} \mathcal{F}^{-0}+c_{02} \mathcal{F}^{-2}+c_{03} \mathcal{F}^{-3}\right), \hat{G}_{1}^{-}=-\mathcal{F}_{1}^{-}+\epsilon d_{11} G^{-1} \\
\hat{G}_{2}^{-}=G_{2}^{-}+\epsilon\left(c_{20} \mathcal{F}^{-0}+c_{22} \mathcal{F}^{-2}+c_{23} \mathcal{F}^{-3}\right), \hat{G}_{3}^{-}=G_{3}^{-}+\epsilon\left(c_{30} \mathcal{F}^{-0}+c_{32} \mathcal{F}^{-2}+c_{33} \mathcal{F}^{-3}\right)(43
\end{gathered}
$$

Substituting expressions for the couplings (40) and the field strengths (43) in relation $\hat{G}_{I}^{-}=$ $\overline{\hat{N}}_{I J} \hat{F}^{-J}$ (any $I=0,1,2,3$ can be used ), we obtain the relation

$$
\mathcal{F}^{-1}=-\frac{\bar{N}_{10}}{\bar{N}_{11}} \hat{\mathcal{F}}^{-0}+\frac{1}{\bar{N}_{11}} \hat{\mathcal{F}}^{-1}-\frac{\bar{N}_{12}}{\bar{N}_{11}} \hat{\mathcal{F}}^{-2}-\frac{\bar{N}_{13}}{\bar{N}_{11}} \hat{\mathcal{F}}^{-3}
$$

which does not contain the constants $d_{11}$ and $c_{i j}$. Here and below the entries with hats refer to the section associated with the heterotic string compactification.

The Kähler potential is invariant under symplectic transformations.

\section{$4 \quad$ Killing spinor equations}

Requiring the supersymmetry variations of gravitino and gaugini to vanish, we obtain a system of Killing spinor equations. To write the supersymmetry transformations, one introduces symplectic invariants $[21,25,26]$

$$
\begin{aligned}
& S_{\mu \nu}=X^{I} \operatorname{Im} N_{I J} \mathcal{F}_{\mu \nu}^{-J}, \\
& T_{\mu \nu}^{-} \equiv 2 i e^{K / 2} S_{\mu \nu}=2 i e^{K / 2} X^{I} \operatorname{Im} N_{I J} \mathcal{F}_{\mu \nu}^{-J}
\end{aligned}
$$

and

$$
G_{\mu \nu}^{-i}=-k^{i \bar{j}} \bar{f} \frac{I}{j} \operatorname{Im} N_{I J} \mathcal{F}_{\mu \nu}^{-J} .
$$

Here $k^{i \bar{j}}$ is the inverse Kähler metric, and $f_{i}^{I}=\left(\partial_{z_{i}}+\frac{1}{2} \partial_{z_{i}} K\right) e^{K / 2} X^{I}$. Supersymmetry transformations of the chiral gravitino $\psi_{\alpha \mu}$ and gaugini $\lambda_{\alpha}^{i}$ are

$$
\begin{array}{r}
\delta \psi_{\alpha \mu}=D_{\mu} \epsilon_{\alpha}-T_{\mu \nu}^{-} \gamma^{\nu} \epsilon_{\alpha \beta} \epsilon^{\beta}, \\
\delta \lambda_{\alpha}^{i}=i \gamma^{\mu} \partial_{\mu} z^{i} \epsilon^{\alpha}+G_{\mu \nu}^{-i} \gamma^{\mu} \gamma^{\nu} \epsilon^{\alpha \beta} \epsilon_{\beta},
\end{array}
$$

where

$$
D_{\mu} \epsilon_{\alpha}=\left(\partial_{\mu}-\frac{1}{4} w_{\mu}^{\hat{a} \hat{b}} \gamma_{\hat{a}} \gamma_{\hat{b}}+\frac{i}{2} Q_{\mu}\right) \epsilon_{\alpha}
$$

Here $w_{\mu}^{\hat{a} \hat{b}}$ and $Q_{\mu}=-\frac{i}{2}\left(\partial_{i} K \partial y_{i}-\partial_{i} K \partial \bar{y}_{i}\right)$ are the spin and Kähler connections; $\hat{a}, \hat{b}, \ldots$ are the tangent space indices, $a, b, \ldots$ are the space-time indices.

The metric of a stationary spherically-symmetric configuration is

$$
d s^{2}=-e^{2 U}\left(d t+w_{i} d x^{i}\right)^{2}+e^{-2 U} d x^{i} d x_{i}
$$


The only non-vanishing components of the spin connection $w_{0}^{\hat{a} \hat{b}}$ are $w_{0}^{\hat{0} \hat{b}}=\frac{1}{2} \partial_{b} e^{2 U}$ and $w_{0}^{\hat{a} \hat{b}}=$ $\frac{1}{2}\left(\partial_{a} w_{b}-\partial_{b} w_{a}\right)$.

We look for a solution of the system of Killing spinor equations with the supersymmetry parameter satisfying the relation $\epsilon^{\alpha}=\gamma_{\hat{0}} \epsilon^{\alpha \beta} \epsilon_{\beta}{ }^{5}$. The $\mu=0$ component of Eq.(47) takes the form

$$
\frac{1}{4} w_{0}^{-\hat{a} \hat{b}} \gamma_{\hat{a}} \gamma_{\hat{b}} \epsilon_{\alpha}+T_{0 n}^{-} e^{\hat{b} n} \gamma_{\hat{b}} \epsilon_{\alpha \beta} \epsilon^{\beta}=0
$$

Here, using antisymmetry of $w_{0}^{\hat{a} \hat{b}}$ in upper indices and chirality of the spinor $\epsilon_{\alpha}$, we transformed $w_{0}^{\hat{a} \hat{b}}$ into $w_{0}^{-\hat{a} \hat{b}}$. Using the relations

$$
G_{\hat{m} \hat{n}}^{-}=i e_{\hat{m} \hat{n} \hat{p} \hat{0}} G^{-\hat{p} \hat{0}}, \quad G_{\mu \nu}^{-} \gamma^{\mu} \gamma^{\nu} \epsilon_{\alpha}=4 G_{\hat{0} \hat{n}}^{-} \gamma^{\hat{0}} \gamma^{\hat{n}} \epsilon_{\alpha}
$$

valid for any self-dual tensor and chiral spinor, Eq.(49) can be rewritten as

$$
\left(w_{0}^{-\hat{0} \hat{b}}-T_{0 n}^{-} e^{\hat{b} n}\right) \gamma_{\hat{b}} \epsilon_{\alpha \beta} \epsilon^{\beta}=0 .
$$

To have a nontrivial solution for the supersymmetry parameter, we require that

$$
w_{0}^{-\hat{0} \hat{b}}-T_{0 n}^{-} e^{\hat{b} n}=0
$$

or, taking the real part of this relation,

$$
\frac{1}{4} \partial_{n} e^{2 U}-e^{U} \operatorname{Re} T_{0 n}^{-}=0
$$

Sufficient condition to have a static metric is $\operatorname{Im} T_{0 n}^{-}=0$.

Using the relations (50), conditions of gaugini supersymmetry transformation to vanish are written as

$$
\left(i \gamma^{n} \partial_{n} z^{i} \gamma^{\hat{0}}+4 G_{\hat{0} \hat{n}}^{-i} \gamma^{\hat{0}} \gamma^{\hat{n}}\right) \epsilon^{\alpha \beta} \epsilon_{\beta}=0 .
$$

Transforming all the $\gamma$-matrices to the tangent space (or world) indices, we find that there is a nontrivial solution provided

$$
i \partial_{n} z^{i}+4 e^{-U} G_{0 n}^{-i}=0 .
$$

Contracting the equation (54) with the function $f_{i}^{I}$ and using the relation of special $N=2$ geometry

$$
k^{i \bar{j}} f_{i}^{I} \bar{f}_{\bar{j}}^{J}=-\frac{1}{2}(\operatorname{Im} N)^{I J}-e^{K} \bar{X}^{I} X^{J},
$$

it is obtained in the form (cf.[27, 28])

$$
i f_{i}^{I} \partial_{n} z^{i}+4 e^{-U}\left(\frac{1}{2} \mathcal{F}_{0 n}^{-I}+e^{K} \bar{X}^{I} S_{0 n}\right)=0
$$

\footnotetext{
${ }^{5}$ More exactly, following [29], one must extract from the supersymmetry parameter the coordinate-dependent factor.
} 
Eq. (55) can be recast to a form which contains the fields $G_{I 0 n}^{-}$and $\bar{F}_{I}$. Introducing $g_{I i}=$ $\left(\partial_{i}+\frac{1}{2} \partial_{i} K\right) e^{K / 2} F_{I}$, contracting Eq.(55) with $\bar{F}_{I}$ and using the identities

$$
\mathcal{F}^{-I} \bar{F}_{I}=G_{I}^{-} \bar{X}^{I}, \quad \bar{F}_{I} f_{i}^{I}=\bar{X}^{I} g_{I i}
$$

which follow from definitions of $f_{i}^{I}$ and $G_{I \mu \nu}^{-}$, we have

$$
i \bar{X}^{I} g_{I i} \partial_{n} z^{i}+4 e^{-U}\left(\frac{1}{2} G_{I 0 n}^{-} \bar{X}^{I}+e^{K} \bar{F}_{I} \bar{X}^{I} S_{0 n}\right)=0 .
$$

Removing the functions $\bar{X}^{I}$, we obtain the symmetric equation

$$
i g_{I i} \partial_{n} z^{i}+4 e^{-U}\left(\frac{1}{2} G_{I 0 n}^{-}+e^{K} \bar{F}_{I} S_{0 n}\right)=0 .
$$

Using the gravitino equation, Eqs.(55) and (56) are presented as (cf. [22])

$$
\begin{gathered}
-2 \mathcal{F}_{0 n}^{-I}=i\left[e^{U} \partial_{n}\left(e^{K / 2} X^{I}\right)-\left(e^{K / 2} \bar{X}^{I}\right) \partial_{n} e^{U}\right]+2 \operatorname{Im} T_{0 n}^{-} e^{K / 2} \bar{X}^{I}-\operatorname{Im}\left(\partial_{i} K \partial_{n} y_{i}\right) e^{K / 2+U} X^{I}, \\
-2 G_{I 0 n}^{-}=i\left[e^{U} \partial_{n}\left(e^{K / 2} F_{I}\right)-\left(e^{K / 2} \bar{F}_{I}\right) \partial_{n} e^{U}\right]+2 \operatorname{Im} T_{0 n}^{-} e^{K / 2} \bar{F}_{I}-\operatorname{Im}\left(\partial_{i} K \partial_{n} y_{i}\right) e^{K / 2+U} F_{I} .
\end{gathered}
$$

Here we used the equality $\partial_{z^{i}} K \partial_{n} z^{i}=\frac{1}{2} \partial_{n} K+i \operatorname{Im}\left(\partial_{y_{i}} K \partial_{n} y_{i}\right)$. Eqs.(57) and (58) are not independent, but one set can be obtained from the other. One can also take some equations from the first set, and the remaining equations from the second. In this paper we shall use the first set of Eqs.(55) or (57). Another choice useful for practical calculations is to take for $I=0,1$ the equations from the first set (57) and for $I=3,4$ from the second (58).

\section{Solution of the tree-level Killing spinor equations}

In this section, to fix notations for the following, we solve the tree-level system of Killing spinor equations for the moduli (cf. [27, 29, 35]). We look for a static solution in the holomorphic section associated with the compactified heterotic string with two magnetic fields $\hat{\mathcal{F}}_{\mu \nu}^{0}$ and $\hat{\mathcal{F}}_{\mu \nu}^{1}$ and two electric fields $\hat{\mathcal{F}}_{\mu \nu}^{2}$ and $\hat{\mathcal{F}}_{\mu \nu}^{3}$. We consider the case of purely real tree-level moduli $y_{i}$ (21), i.e. $a_{i}=0$. The moduli in the heterotic holomorphic section are expressed via the moduli in the section with the prepotential as [12]

$$
\left(\hat{X}^{I}, \hat{F}_{I}\right)=\left(1, y_{2} y_{3}, i y_{2}, i y_{3} ;-i y_{1} y_{2} y_{3},-i y_{1}, y_{1} y_{3}-\epsilon h_{2}, y_{1} y_{2}-\epsilon h_{3}\right) .
$$

Solving the Maxwell equations and Bianchi identities we obtain the tree-level magnetic

$$
\hat{\mathcal{F}}_{0 n}^{-0}=\frac{i}{2} \frac{\hat{P}^{0}}{\sqrt{-g^{\prime}}} \frac{x^{n}}{r}=\frac{i}{2} e^{2 U} \hat{P}^{0} \frac{x^{n}}{r^{3}}, \quad \hat{\mathcal{F}}_{0 n}^{-1}=\frac{i}{2} \frac{\hat{P}^{1}}{\sqrt{-g^{\prime}}} \frac{x^{n}}{r}=\frac{i}{2} e^{2 U} \hat{P}^{1} \frac{x^{n}}{r^{3}}
$$

and electric

$$
\hat{\mathcal{F}}_{0 n}^{-2}=-\frac{\hat{Q}_{2}}{2 \sqrt{-g^{\prime}} I m \hat{N}_{22}} \frac{x^{n}}{r}, \quad \hat{\mathcal{F}}_{0 n}^{-3}=-\frac{\hat{Q}_{3}}{\sqrt{-g^{\prime}} I m \hat{N}_{33}} \frac{x^{n}}{r}
$$


field strengths, where $\sqrt{-g^{\prime}}=r^{2} e^{-2 U}$ and the gauge couplings are

$$
\hat{N}_{00}=-i y_{1} y_{2} y_{3}, \quad \hat{N}_{11}=-i \frac{y_{1}}{y_{2} y_{3}}, \quad \hat{N}_{22}=-i \frac{y_{1} y_{3}}{y_{2}}, \quad \hat{N}_{33}=-i \frac{y_{1} y_{2}}{y_{3}} .
$$

In the case of real moduli $y_{i}$, comparing with the action (1), we have

$$
\operatorname{Im}\left(\hat{N}_{00}, \hat{N}_{11}, \hat{N}_{22}, \hat{N}_{33}\right)=-e^{-\phi}\left(G_{11}, G^{11}, G_{22}, G^{22}\right)
$$

(we remind that hatted expressions refer to the section associated with the heterotic string compactification). The charges $\sqrt{8} \hat{P}^{I}$ and $\sqrt{8} \hat{Q}_{I}$ are constrained to lie on an even self-dual lattice [19]. The tree-level Kähler potential is

$$
K=-\ln 8 y_{1} y_{2} y_{3}
$$

For the symplectic invariant $S_{0 n}(45)$ we obtain

$$
\begin{aligned}
S_{0 n} & =\left(\operatorname{Im} \hat{N}_{00} \hat{\mathcal{F}}_{0 n}^{-0}+y_{2} y_{3} \operatorname{Im} \hat{N}_{11} \hat{\mathcal{F}}_{0 n}^{-1}+i y_{2} \operatorname{Im} \hat{N}_{22} \hat{\mathcal{F}}_{0 n}^{-2}+i y_{3} \operatorname{Im} \hat{N}_{33} \hat{\mathcal{F}}_{0 n}^{-3}\right) \\
& =-i \frac{y_{1} y_{2} y_{3}}{2}\left(\hat{P}^{0}+\frac{\hat{P}^{1}}{y_{2} y_{3}}+\frac{\hat{Q}_{2}}{y_{1} y_{3}}+\frac{\hat{Q}_{3}}{y_{1} y_{2}}\right) e^{2 U} \frac{x^{n}}{r^{3}} .
\end{aligned}
$$

Gravitini Eq.(52) takes the form

$$
\frac{1}{4} \partial_{n} e^{2 U}-\left(\frac{y_{1} y_{2} y_{3}}{8}\right)^{1 / 2} e^{3 U}\left(\hat{P}^{0}+\frac{\hat{P}^{1}}{y_{2} y_{3}}+\frac{\hat{Q}_{2}}{y_{1} y_{3}}+\frac{\hat{Q}_{3}}{y_{1} y_{2}}\right) \frac{x^{n}}{r^{3}}=0 .
$$

The tree-level gaugini equations (55) written in the section associated with the prepotential are

$$
\begin{aligned}
& I=0: \quad \frac{i e^{K / 2}}{2} \partial_{n} \ln y_{1} y_{2} y_{3}-4 e^{-U}\left(\frac{1}{2} \mathcal{F}_{0 n}^{-0}+e^{K} S_{0 n}\right)=0 \\
& I=1: \quad \frac{y_{1} e^{K / 2}}{2} \partial_{n} \ln \frac{y_{2} y_{3}}{y_{1}}+4 e^{-U}\left(\frac{\hat{\mathcal{F}}_{0 n}^{-1}}{2 \bar{N}_{11}}-i y_{1} e^{K} S_{0 n}\right)=0 \\
& I=2: \quad \frac{y_{2} e^{K / 2}}{2} \partial_{n} \ln \frac{y_{1} y_{3}}{y_{2}}+4 e^{-U}\left(\frac{1}{2} \mathcal{F}_{0 n}^{-2}-i y_{2} e^{K} S_{0 n}\right)=0 \\
& I=3: \quad \frac{y_{3} e^{K / 2}}{2} \partial_{n} \ln \frac{y_{1} y_{2}}{y_{3}}+4 e^{-U}\left(\frac{1}{2} \mathcal{F}_{0 n}^{-3}-i y_{3} e^{K} S_{0 n}\right)=0
\end{aligned}
$$

The field strengths in the section with the prepotential are defined from those (60) and (61) by using (43) and (44).

In the following, we consider a particular extremal dyonic solution of the Eqs.(64) with arbitrary constant moduli $y_{2}$ and $y_{3}$ and with the charges subject to relations

$$
\hat{P}^{0}=\frac{\hat{P}^{1}}{y_{2} y_{3}}, \quad \hat{Q}_{2} y_{2}=\hat{Q}_{3} y_{3} .
$$


Four gaugini equations reduce to one equation. The system of the gravitini and gaugini equations is

$$
\begin{aligned}
& \partial_{n} e^{2 U}-\left(8 y_{1} y_{2} y_{3}\right)^{1 / 2} e^{3 U}\left(\hat{P}^{0}+\frac{\hat{Q}_{2}}{y_{1} y_{3}}\right) \frac{x^{n}}{r^{3}}=0 \\
& \partial_{n} \ln y_{1}-\left(8 y_{1} y_{2} y_{3}\right)^{1 / 2} e^{U}\left(\hat{P}^{0}-\frac{\hat{Q}_{2}}{y_{1} y_{3}}\right) \frac{x^{n}}{r^{3}}=0 .
\end{aligned}
$$

Introducing the charges $P$ and $Q$ as

$$
P=\sqrt{8 y_{2} y_{3}} \hat{P}^{0}, \quad Q=\hat{Q}_{2} \sqrt{\frac{8 y_{2}}{y_{3}}},
$$

for the metric and dilaton we obtain

$$
e^{-2 U}=\frac{(P+r)(Q+r)}{r^{2}}, \quad y_{1}^{-1}=e^{\phi}=\frac{P+r}{Q+r} \equiv f_{0}
$$

which is a particular case of a general dyonic BPS saturated solution [31, 32] The components of the metric $G_{m n}$ are

$$
G_{11}=y_{2} y_{3}, \quad G_{22}=\frac{y_{2}}{y_{3}}
$$

The factor $\sqrt{8}$ appears because of different normalizations of the gauge terms in the actions (3) and (19).

The charges $\hat{P}^{0,1}$ and $\hat{Q}_{2,3}$ are expressed via the charges $P$ and $Q$ as

$$
\hat{P}^{0}=\frac{P e^{-\gamma_{0}}}{\sqrt{8}}, \quad \hat{P}^{1}=\frac{P e^{\gamma_{0}}}{\sqrt{8}}, \quad \hat{Q}_{2}=\frac{Q e^{-\sigma_{0}}}{\sqrt{8}}, \quad \hat{Q}_{3}=\frac{Q e^{\sigma_{0}}}{\sqrt{8}} .
$$

Here $\gamma_{0}$ and $\sigma_{0}$ are the tree-level values of the moduli $\gamma$ and $\sigma$ parameterizing the metric of the two torus (23).

\section{Solution of the system of the loop-corrected Maxwell and Killing spinor equations}

Our next aim is to solve the system of Maxwell and Killing spinor equations for the loopcorrected metric and moduli using as the input the tree-level solution (69). We look for a solution in the first order in the string coupling constant. The loop corrections to the gauge coupling constants are calculated by substituting the tree-level moduli. The terms which depend on the constant tree-level moduli $y_{2}$ and $y_{3}$ are independent of coordinates. Dependence on coordinates enters through the modulus $y_{1}=f_{0}^{-1}$ and the metric.

Let us introduce notations for the loop-corrected metric and moduli. The functions $\phi, \gamma$ and $\sigma$ which enter the moduli (23) are split into the tree-level $\phi_{0}, \gamma_{0}$ and $\sigma_{0}$ and first-order parts in string coupling $\phi_{1}, \gamma_{1}$ and $\sigma_{1}: \phi=\phi_{0}+\epsilon \phi_{1}$, etc. The function $U$ which enters the metric will be written as $2 U_{0}+\epsilon u_{1}$. The tree-level expressions for the functions $U_{0}$ and $\phi_{0}$ are given by (69), where $\gamma_{0}$ and $\sigma_{0}$ are arbitrary constants. 


\subsection{Electric field strengths}

First let us solve the Maxwell equations (in the heterotic holomorphic section) which can be rewritten in the form

$$
\partial_{\mu}\left(\sqrt{-g} \operatorname{Im} \hat{N}_{I J} \hat{\mathcal{F}}^{J}+\operatorname{Re} \hat{N}_{I J}^{*} \hat{\mathcal{F}}^{J}\right)^{\mu \nu}=0 .
$$

With the accuracy of the terms of the first order in string coupling $\epsilon$, Eqs.(71) written in spherical coordinates are

$$
\begin{gathered}
I=0: \partial_{r}\left[\sqrt{-g}\left(\operatorname{Im} \hat{N}_{00} \hat{\mathcal{F}}^{0}+\operatorname{Im} \hat{N}_{02} \hat{\mathcal{F}}^{2}+\operatorname{Im} \hat{N}_{03} \hat{\mathcal{F}}^{3}\right)+\operatorname{Re} \hat{N}_{00} * \hat{\mathcal{F}}^{0}+\operatorname{Re} \hat{N}_{01}{ }^{*} \hat{\mathcal{F}}^{1}\right]^{0 r}=0 \\
I=1: \partial_{r}\left[\sqrt{-g}\left(\operatorname{Im} \hat{N}_{11} \hat{\mathcal{F}}^{1}+\operatorname{Im} \hat{N}_{12} \hat{\mathcal{F}}^{2}+\operatorname{Im} \hat{N}_{13} \hat{\mathcal{F}}^{3}\right)+\operatorname{Re} \hat{N}_{10}{ }^{*} \hat{\mathcal{F}}^{0}+R e \hat{N}_{11}{ }^{*} \hat{\mathcal{F}}^{1}\right]^{0 r}=0 \\
I=2: \quad \partial_{r}\left[\sqrt{-g}\left(\operatorname{Im} \hat{N}_{22} \hat{\mathcal{F}}^{2}+\operatorname{Im} \hat{N}_{23} \hat{\mathcal{F}}^{3}\right)+\operatorname{Re} \hat{N}_{20}{ }^{*} \hat{\mathcal{F}}^{0}+\operatorname{Re} \hat{N}_{21}{ }^{*} \hat{\mathcal{F}}^{1}\right]^{0 r}=0 \\
I=3: \quad \partial_{r}\left[\sqrt{-g}\left(\operatorname{Im}_{33} \hat{\mathcal{F}}^{3}+\operatorname{Im}_{32} \hat{\mathcal{F}}^{2}\right)+\operatorname{Re} \hat{N}_{30}{ }^{*} \hat{\mathcal{F}}^{0}+\operatorname{Re}_{31}{ }^{*} \hat{\mathcal{F}}^{1}\right]^{0 r}=0
\end{gathered}
$$

Only the diagonal gauge couplings $\hat{N}_{I I}$ contain terms of zero order in string coupling. The field strengths $\hat{\mathcal{F}}^{0,10 r}$, absent at the tree level, are of the first order in the string coupling. Substituting the tree-level field strengths (60) and (61) in Eqs.(72) and (73), we have

$$
\begin{aligned}
\hat{\mathcal{F}}^{00 r} & =\frac{\epsilon \hat{q}_{0}-\operatorname{Re} \hat{N}_{00} \hat{P}^{0}-\operatorname{Re} \hat{N}_{01} \hat{P}^{1}-\hat{Q}_{2} \frac{\operatorname{Im} \hat{N}_{02}}{\operatorname{Im} \hat{N}_{22}}-\hat{Q}_{3} \frac{\operatorname{Im} \hat{N}_{03}}{\operatorname{Im} \hat{N}_{33}}}{\sqrt{-g^{\prime}} \operatorname{Im} \hat{N}_{00}}, \\
\hat{\mathcal{F}}^{10 r} & =\frac{\epsilon \hat{q}_{1}-\operatorname{Re} \hat{N}_{10} \hat{P}^{0}-\operatorname{Re} \hat{N}_{11} \hat{P}^{1}-\hat{Q}_{2} \frac{\operatorname{Im} \hat{N}_{12}}{\operatorname{Im} \hat{N}_{22}}-\hat{Q}_{3} \frac{\operatorname{Im} \hat{N}_{13}}{\operatorname{Im} \hat{N}_{33}}}{\sqrt{-g^{\prime}} \operatorname{Im} \hat{N}_{11}},
\end{aligned}
$$

where $\hat{q}_{0,1}$ are arbitrary constants which have a meaning of electric charges of the first order in string coupling. Since the numerators are of the first order in string coupling, denominators are taken in the leading order.

In the case of constant moduli $y_{2}$ and $y_{3}$ the ambiguities of the couplings in the matrix (41) which are real enter the topological terms in the action and do not affect the equations of motion. The imaginary ambiguities $\delta \hat{N}_{0 a}$ and $\delta \hat{N}_{1 a}$ of the gauge couplings appear in the Maxwell equations. Substituting explicit expressions for the couplings, we obtain

$$
\begin{aligned}
\hat{\mathcal{F}}^{00 r} & =\epsilon \frac{\hat{q}_{0}-a_{1} \hat{P}^{1}-\hat{Q}_{2}\left(a_{2}+\left(b-2 b_{2} y_{2}\right) / y_{1} y_{3}\right)-\hat{Q}_{3}\left(a_{3}+\left(b-2 b_{3} y_{3}\right) / y_{1} y_{2}\right)}{\sqrt{-g^{\prime}} I m \hat{N}_{00}} \\
\hat{\mathcal{F}}^{10 r} & =\epsilon \frac{\hat{q}_{1}-a_{1} \hat{P}^{0}+\hat{Q}_{2}\left(a_{3} / y_{3}^{2}-\left(2 b_{2} y_{2}-b\right) / y^{3} y_{3}\right)+\hat{Q}_{3}\left(a_{2} / y_{2}^{2}-\left(2 b_{3} y_{3}-b\right) / y^{3} y_{2}\right)}{\sqrt{-g^{\prime}} \operatorname{Im} \hat{N}_{11}}
\end{aligned}
$$

For the case we consider with the charges subjected to conditions (70) the ambiguities in the strengths (77) cancel, and the remaining expressions are unambiguous.

The Eqs.(74) and (75) yield

$$
\begin{aligned}
\hat{\mathcal{F}}^{20 r} & =\frac{\hat{Q}_{2}-\operatorname{Re} \hat{N}_{20} \hat{P}^{0}-\operatorname{Re} \hat{N}_{21} \hat{P}^{1}-\frac{\operatorname{Im} \hat{N}_{23}}{\operatorname{Im} \hat{N}_{33}} \hat{Q}_{3}}{\sqrt{-g^{\prime}} \operatorname{Im} \hat{N}_{22}} \\
\hat{\mathcal{F}}^{30 r} & =\frac{\hat{Q}_{3}-\operatorname{Re} \hat{N}_{30} \hat{P}^{0}-\operatorname{Re} \hat{N}_{31} \hat{P}^{1}-\frac{\operatorname{Im} \hat{N}_{32}}{\operatorname{Im} \hat{N}_{22}} \hat{Q}_{2}}{\sqrt{-g^{\prime}} \operatorname{Im} \hat{N}_{33}} .
\end{aligned}
$$


At this point there are two options. Either we can allow for appearance of the fields absent at the tree level with the charges of the first order in string coupling, or we can require that the loop-corrected solution, as the tree level one, contains two electric and two magnetic fields. Since magnetic fields ${ }^{*} \hat{\mathcal{F}}^{2,3}$ with the charges $\hat{p}_{2,3}$ of the first order in string coupling enter the Maxwell equations multiplied by the non-diagonal gauge couplings $N_{I J}$ which are of the first order in string coupling and thus cannot be fixed with the accuracy of the first order in string coupling, we set the charges $p_{2,3}$ equal to zero, but retain the electric charges $\hat{q}_{0}$ and $\hat{q}_{1}$.

In the section associated with the prepotential, the field strengths can be obtained either by direct solution of the system of Maxwell equations and Bianchi identities or by using relations (43) and (44).

Using solutions for the field strengths (76) and (78), in the first order in the string coupling constant, we calculate the symplectic invariant $S_{0 n}(45)$ as

$$
\begin{aligned}
& S_{0 n}=\left\{\left[\hat{P}^{0}\left(\operatorname{Im} N_{00}+y_{i} \operatorname{Re} N_{i 0}\right)-\hat{P}^{1} y_{1}-\left(\hat{Q}_{2} y_{2}+\hat{Q}_{3} y_{3}\right)\right]\right. \\
& \left.\left.-i \epsilon\left[\hat{P}^{0}\left(a_{1} y_{2} y_{3}+a_{2} y_{1} y_{3}+a_{3} y_{1} y_{2}\right)+\hat{P}^{1} a_{1}+a_{a} \hat{Q}_{a}-\hat{q}_{0}-\hat{q}_{1} y_{2} y_{3}\right)\right]\right\} \frac{i}{2} e^{2 U} \frac{x^{n}}{r^{3}}
\end{aligned}
$$

Only the the couplings $N_{00}$ and $N_{0 i}, i=1,2,3$ enter the expression (79). Substituting the loop-corrected gauge couplings (30), we obtain

$$
\operatorname{Im} N_{00}+y_{i} \operatorname{Re} N_{i 0}=-y^{3}-\epsilon\left(2 v+h_{a} y_{a}\right) .
$$

All the terms containing second derivatives of the loop correction to the prepotential have canceled.

Substituting in (79) the expression (80), we finally have

$$
\begin{aligned}
& S_{0 n}=\left\{\left[-\hat{P}^{0}\left(y^{3}+\epsilon\left(2 v+h_{a} y_{a}\right)\right)-\hat{P}^{1} y_{1}-\hat{Q}_{a} y_{a}\right]\right. \\
& \left.\left.-i \epsilon\left[\hat{P}^{0}\left(a_{1} y_{2} y_{3}+a_{2} y_{1} y_{3}+a_{3} y_{1} y_{2}\right)+\hat{P}^{1} a_{1}+\hat{Q}_{a} a_{a}-\hat{q}_{0}-\hat{q}_{1} y_{2} y_{3}\right)\right]\right\} \frac{i}{2} e^{2 U} \frac{x^{n}}{r^{3}}
\end{aligned}
$$

Because the Killing spinor equations (54) are linear in derivatives of the moduli $\partial z^{i}$, the equations for the real parts of the moduli decouple from imaginary parts. Since Killing spinor equations from which are determined the loop corrections to the dilaton $\phi$ and metric component $e^{2 U}$ contain only the real parts of the moduli, to determine these corrections we can ignore the imaginary parts of the moduli. The imaginary parts of the moduli are of the first order in string coupling, and are determined from the imaginary parts of the Killing spinor equations which contain the real parts only in the main order ${ }^{6}$.

\subsection{Loop-corrected gravitini Killing spinor equation}

Expanding the Kähler potential (27) to the first order in string coupling, we obtain

$$
e^{K}=\frac{f_{0} e^{-2 \gamma_{0}}}{8}\left[1+\epsilon\left(\phi_{1}-2 \gamma_{1}\right)\right] \text {. }
$$

\footnotetext{
${ }^{6}$ To have a static metric, we must choose a solution for which $\operatorname{Im} T_{0 n}^{-}=0$ (see. Sect.3).
} 
Using this expression for the Kähler potential and calculating to the first order in string coupling the real part of the symplectic invariant $T_{0 n}^{-}=2 i e^{K / 2} S_{0 n}$ which enters the gravitini equation (52) we have

$$
T_{0 n}^{-}=\frac{f_{0}^{-1 / 2}}{4}\left[P\left(1-\epsilon \frac{\phi_{1}}{2}+\epsilon H f_{0}\right)+Q f_{0}\left(1+\epsilon \frac{\phi_{1}}{2}\right)\right] e^{2 U} \frac{x^{n}}{r^{3}}
$$

Here we introduced

$$
H=\frac{h}{2} e^{-2 \gamma_{0}}
$$

All the factors multiplying the expressions of the first order in string coupling are taken in the main order. It is convenient to introduce the functions $q^{\prime}$ and $l^{\prime}$ as

$$
q^{\prime}=\frac{f_{0}{ }^{\prime}}{f_{0}}=\frac{Q-P}{(r+P)(r+Q)}, \quad l^{\prime}=2 U_{0}^{\prime}=\frac{2 P Q+P r+Q r}{r(r+P)(r+Q)} .
$$

Now the gravitino equation (52) takes the form

$$
\frac{u_{1}^{\prime}}{l^{\prime}}-\frac{u_{1}}{2}+\frac{\phi_{1}}{2} \frac{P-Q f_{0}}{P+Q f_{0}}-\frac{P H f_{0}}{P+Q f_{0}}=0 .
$$

The leading-order terms have canceled due to the Eqs.(64).

\subsection{Loop-corrected gaugini Killing spinor equation}

Let us solve the gaugini Killing spinor equations in the form (55). In the first order in string coupling, the combinations $f_{i}^{I} \partial_{n} z^{i}$ which enter the equations (54) are

$$
\begin{aligned}
f_{i}^{0} \partial_{n} z^{i} & =-\frac{1}{2} e^{K / 2}\left(1-\epsilon \frac{V}{2 y_{1}}\right) \partial_{n} \ln y_{1} y_{2} y_{3} \\
f_{i}^{I} \partial_{n} z^{i} & =i y_{i}\left(B_{n}^{0}+e^{K / 2} \partial_{n} \ln y_{i}\right) . \quad i=1,2,3,
\end{aligned}
$$

With the accuracy up to the terms of order $O(\epsilon)$, the expression $f_{i}^{0} \partial_{n} z^{i}$ is

$$
f_{i}^{0} \partial_{n} z^{i}=\frac{q^{\prime} f_{0}{ }^{1 / 2} e^{-\gamma_{0}}}{2 \sqrt{8}}\left[1+\epsilon\left(\frac{\phi_{1}{ }^{\prime}-2 \gamma_{1}{ }^{\prime}}{q^{\prime}}+\frac{\phi_{1}-2 \gamma_{1}}{2}\right)\right] \frac{x^{n}}{r} .
$$

Substituting the explicit expressions for the gauge couplings, we obtain the combination $\frac{1}{2} \mathcal{F}_{0 n}^{-0}+$ $e^{K} S_{0 n}$ in Eq.(55) as

$$
\frac{1}{2} \mathcal{F}_{0 n}^{-0}+e^{K} S_{0 n}=\frac{e^{-\gamma_{0}}}{4 \sqrt{8}}\left[P\left(1+\epsilon\left(\gamma_{1}-H f_{0}\right)\right)-Q f_{0}\left(1+\epsilon\left(\phi_{1}-\gamma_{1}\right)\right)\right] \frac{i}{2} e^{2 U} \frac{x^{n}}{r^{3}}
$$

Using Eqs.(89) and (88), we obtain the $I=0$ gaugini equation in the form

$$
\frac{\phi_{1}^{\prime}-2 \gamma_{1}^{\prime}}{q^{\prime}}+\frac{\phi_{1}}{2} \frac{P+Q f_{0}}{P-Q f_{0}}-\frac{u_{1}}{2}-2 \gamma_{1} \frac{P}{P-Q f_{0}}+\frac{P H f_{0}}{P-Q f_{0}}=0
$$


Following the same steps, we obtain the $I=1$ gaugini equation as

$$
\frac{\phi_{1}^{\prime}+2 \gamma_{1}^{\prime}}{q^{\prime}}+\frac{\phi_{1}}{2} \frac{P+Q f_{0}}{P-Q f_{0}}-\frac{u_{1}}{2}+2 \gamma_{1} \frac{P}{P-Q f_{0}}+\frac{P H f_{0}}{P-Q f_{0}}=0 .
$$

Eqs.(90) and (91) split into the pair of equations

$$
\frac{\phi_{1}^{\prime}}{q^{\prime}}+\frac{\phi_{1}}{2} \frac{P+Q f_{0}}{P-Q f_{0}}-\frac{u_{1}}{2}+\frac{P H f_{0}}{P-Q f_{0}}=0
$$

and

$$
\frac{\gamma_{1}^{\prime}}{q^{\prime}}+\gamma_{1} \frac{P}{P-Q f_{0}}=0
$$

Using the expressions (85) for $q^{\prime}$ and (69) for $f_{0}$, we rewrite Eq.(93) as

$$
\gamma_{1}^{\prime}-\gamma_{1} \frac{P}{r(r+P)}=0
$$

Combining Eqs.(55) with $I=0$ and $I=2$ (the same with $I=3$ ), and using the expressions (87) for $B_{n}^{I}$, we have

$$
i e^{K / 2} \frac{\partial_{n} y_{2}}{y_{2}}+4 e^{-U}\left(\frac{\mathcal{F}_{0 n}^{-2}}{2 i y_{2}}-\frac{1}{2} \mathcal{F}_{0 n}^{-0}-2 e^{K} S_{0 n}\right)=0 .
$$

Since the tree-level moduli $y_{2}$ and $y_{3}$ are constants, $\partial_{n} y_{2}$ and $\partial_{n} y_{3}$ are of the first order in string coupling. The combination $\left(\frac{\mathcal{F}_{0 n}^{-2}}{2 i y_{2}}-\frac{1}{2} \mathcal{F}_{0 n}^{-0}-2 e^{K} S_{0 n}\right)$ is also of the first order in string coupling. Substituting in (95) (the same with $I=3$ ) the explicit expressions, we arrive at

$$
\begin{aligned}
& \gamma_{1}^{\prime}+\sigma_{1}{ }^{\prime}+\frac{\left(f_{0}\right)^{-1 / 2} e^{U_{0}}}{r^{2}}\left[P\left(\left(L-L_{2}\right) f_{0}-\gamma_{1}\right)-Q f_{0} \sigma_{1}\right]=0 \\
& \gamma_{1}^{\prime}-\sigma_{1}{ }^{\prime}+\frac{\left(f_{0}\right)^{-1 / 2} e^{U_{0}}}{r^{2}}\left[P\left(\left(L-L_{3}\right) f_{0}-\gamma_{1}\right)+Q f_{0} \sigma_{1}\right]=0 .
\end{aligned}
$$

Here we introduced

$$
L_{2}=\frac{h_{2}}{y_{3}}, \quad L_{3}=\frac{h_{3}}{y_{2}}, \quad L=\frac{L_{2}+L_{3}}{2} .
$$

\subsection{Loop corrections to the moduli and metric}

Combining Eqs.(96) we obtain

$$
\begin{aligned}
& \gamma_{1}^{\prime}-\gamma_{1} \frac{P}{r(r+P)}=0 \\
& \sigma_{1}^{\prime}-\frac{f_{0}^{1 / 2} e^{U_{0}}}{r^{2}}\left[\frac{1}{2} P\left(L_{3}-L_{2}\right)+Q \sigma_{1}\right]=0 .
\end{aligned}
$$


The first Eq.(98) is identical to Eq.(94). Thus, although the number of equations exceeds the number of variables, the system of equations is consistent. Substituting explicit expressions (69) for $f_{0}$ and $e^{U_{0}}$, we obtain the equation for $\sigma_{1}$ in the form

$$
\sigma_{1}{ }^{\prime}-\frac{1}{r(Q+r)}\left[\frac{P}{2}\left(L_{2}-L_{3}\right)+Q \sigma_{1}\right]=0 .
$$

Solving equations for the loop corrections to the moduli, we obtain

$$
\begin{aligned}
& \gamma_{1}=C_{1} \frac{r}{r+P} \\
& \sigma_{1}=C_{2} \frac{r}{r+Q}-\frac{\left(L_{2}-L_{3}\right)}{2} \frac{P}{r+Q}
\end{aligned}
$$

where $C_{1}$ and $C_{2}$ are arbitrary constants.

Let us solve the system of equations (86) and (92) for the loop corrections to the metric and dilaton. Using the relation

$$
l^{\prime}=-\frac{P+Q f_{0}}{P-Q f_{0}} q^{\prime},
$$

we transform the system to the form

$$
\begin{aligned}
& \phi_{1}^{\prime}-\frac{u_{1}}{2} q^{\prime}-\frac{\phi_{1}}{2} l^{\prime}+\frac{P H f_{0}{ }^{\prime}}{P-Q f_{0}}=0, \\
& u_{1}^{\prime}-\frac{u_{1}}{2} l^{\prime}-\frac{\phi_{1}}{2} q^{\prime}+\frac{P H f_{0}{ }^{\prime}}{P-Q f_{0}}=0 .
\end{aligned}
$$

We solve the system with the boundary condition that $u_{1}$ and $\phi_{1}$ vanish in the limit $r=\infty$, i.e. the loop-corrected metric and dilaton are asymptotic to the Lorentzian metric and constant dilaton equal to unity. Adding and subtracting Eqs.(102), we obtain two separate equations for $u_{1} \pm \phi_{1}$ which are straightforwardly solved as

$$
u_{1}=\phi_{1}=-\frac{P H}{r+Q},
$$

where $H=\operatorname{Re} h(T, U) / 2 T U$. Here $h$ is the loop correction to the prepotential, $y_{2}=T, y_{3}=U$ are the standard (real) moduli (22) calculated by substituting the tree-level dyonic solution.

To be concrete, let us consider the explicit form of the prepotential which was calculated in [17] for the case of unbroken gauge group is $\left[E_{8} \times E_{7} \times U(1)^{2}\right]_{L} \times U(1)_{R}^{2}$. The loop correction is of the form

$$
\begin{array}{rlrl}
h(T, U) & =R e h-i \frac{U^{3}}{12 \pi}+\delta h_{+}, & T_{2}>U_{2}, \\
& =R e h-i \frac{T^{3}}{12 \pi}+\delta h_{-}, \quad U_{2}>T_{2},
\end{array}
$$

where $R e h$ is negative and $\delta h_{ \pm}$are ambiguities of the prepotential which are quadratic polynomials in T, U.TU with purely imaginary coefficients discussed above. 
Since $R e h<0$, the function $H$ is negative and the loop corrections (103) to the metric and dilaton are positive.

From the expression for the loop-corrected metric

$$
e^{2 U}=\frac{r^{2}}{(r+P)(r+Q)}\left(1-\epsilon \frac{P H}{r+Q}\right)
$$

follows that its domain of validity is $1>\epsilon \frac{P H}{r+Q}$. For $Q>|\epsilon P H|(105)$ is valid for all $r$.

In magnetic case, for the purely magnetic solution of the equations of motion with loop corrections the metric is

$$
e^{2 U}=\frac{r}{r+P}\left(1-\epsilon \frac{P H}{r}\right)
$$

with the range of validity $r>\epsilon|H P|^{7}$. At the same time, the in-put tree-level solution is meaningful in the region where the effective string coupling constant $\epsilon e^{\phi}$ is small, $\epsilon\left(1+\frac{P}{r}\right)<1$, or $r>\epsilon P$. If we extrapolate both the tree-level and the loop-corrected solutions in the broader region, up to the origin, we see that the loop correction becomes dominant in this region. The metric (106) can be considered as the first term in the expansion of the function $\frac{r+|\epsilon P H|}{r+P}$ which can be interpreted as a component of the metric with the smeared singularity at the origin.

\section{BPS mass and asymptotics of the loop-corrected solu- tion}

BPS condition relating the mass of a solution with the central charge of the $N=2$ supersymmetry algebra should retain its form in perturbation theory provided the perturbation theory does not violate supersymmetry. The BPS spectrum of $N=2$ supersymmetric theory is given by $[21]$

$$
M_{B P S}=\left|Z_{\infty}\right|=e^{K / 2}\left|n_{I} X^{I}-m^{I} F_{I}\right|_{\infty},
$$

$n_{I}$ and $m^{I}$ are integers proportional to electric and magnetic charges of the fields (25) gauging the group $U(1)^{4}$. Subscript $\infty$ indicates that the equality is valid at spatial infinity.

Equality of the ADM and BPS masses of a BPS-saturated solution can be seen from the Nester construction in which both masses are expressed via the asymptotics of the function (cf. $[11,33])$

$$
T_{\mu \nu}^{-}=e^{K / 2}\left(F_{I} \mathcal{F}_{\mu \nu}^{I}-X^{I} G_{I \mu \nu}\right),
$$

where $G_{I \mu \nu}=\operatorname{Re} N_{I J} \mathcal{F}_{\mu \nu}^{J}-\operatorname{Im} N_{I J}{ }^{*} \mathcal{F}_{\mu \nu}^{J}$. Expression (107) is the integrated asymptotic form of (108).

\footnotetext{
${ }^{7}$ Generically, loop-corrected solution for magnetic black hole contains also electric charges of the first order in string coupling. In this case in (106) appears the expression $\epsilon(P H+Q)$, where $Q$ is a combination of electric charges. This solution cannot be obtained as the limit $Q \rightarrow 0$ of the dyonic solution, since the limits $r=0$ and $Q=0$ of the function $f_{0}$ and other expressions are not interchangeable. Since these functions appear in the differential equations, one must start from the beginning from the purely magnetic solution.
} 
At the the tree-level, the BPS mass of dyon with two electric and two magnetic $m^{2}, m^{3}$ and two electric charges $n_{0}, n_{1}$ calculated in the heterotic holomorphic section (59) by using the formula (107) reads

$$
M_{B P S}=\frac{1}{\sqrt{8 e^{2 \gamma_{0}}}}\left|n_{0}+n_{1} e^{2 \gamma_{0}}+m^{2} e^{\gamma_{0}-\sigma_{0}}+m^{3} e^{\gamma_{0}+\sigma_{0}}\right|
$$

Setting $\left(n_{0}, n_{1}, m^{2}, m^{3}\right)=\left(\hat{P}^{1}, \hat{P}^{0}, \hat{Q}^{3}, \hat{Q}^{2}\right)$, we obtain (109) as

$$
M_{B P S}=\frac{1}{\sqrt{8}}\left|\hat{P}^{0} e^{\gamma_{0}}+\hat{P}^{1} e^{-\gamma_{0}}+\hat{Q}_{3} e^{-\sigma_{0}}+\hat{Q}_{2} e^{\sigma_{0}}\right|=\frac{1}{4}(P+Q),
$$

where for the second equality we used (70). Here we used the fact that the dilaton is normalized at the infinity to unity: $\left.y_{1}\right|_{\infty}=1$.

On the other hand, calculating the ADM mass from the asymptotics of the metric, $e^{2 U}=$ $1-\frac{(P+Q)}{r}+O\left(r^{-2}\right)$, we have $M_{A D M}=2(P+Q)$. Up to a normalization factor 8 due to different respective normalization of the Einstein and gauge terms in the actions (17) and (19), the ADM and the BPS masses coincide.

At the one-loop level, the BPS mass can be obtained either by using (108), with the loopcorrected (exact, because in $N=2$ supersymmetric theory prepotential receives only oneloop correction) period vector $\left(\hat{X}^{I}, \hat{F}_{I}\right)$, or from from the asymptotics of the loop-corrected symplectic invariant $T_{0 n}^{-}$(83) which enters the Killing spinor equation (52). We have

$$
M_{B P S}=e^{K / 2}\left[\hat{P}^{0}\left(y^{3}+\epsilon\left(2 v+h_{a} y_{a}\right)\right)+\hat{P}^{1} y_{1}+\hat{Q}_{a} y_{a}\right]_{\infty}+O\left(\epsilon^{2}\right),
$$

or,

$$
M_{B P S}=\frac{1}{4}[P(1+\epsilon H)+Q] .
$$

The ADM mass is obtained from the asymptotic form of the metric

$$
\left.e^{2 U}\right|_{\infty}=\left.e^{2 U_{0}}\left(1+\epsilon u_{1}\right)\right|_{\infty}
$$

In the limit $r \rightarrow \infty$ we obtain the asymptotics of the correction to the metric (103)

$$
\left.u_{1}\right|_{r \rightarrow \infty}=-\frac{P H}{r}
$$

Taking into account the normalization factor 8, we find that the loop-corrected BPS and ADM masses are equal to each other.

\section{Equations for axions}

Let us discuss of the equations for the imaginary parts $a_{i}$ of the moduli $y_{i}$.

Convoluting the Eq.(54) with the metric $k_{i \bar{j}}$ (summation over $i$ ), we have

$$
k_{\overline{j i}} \partial_{n}\left(R e y_{i}+i \epsilon a_{i}\right)+4 e^{-U} \bar{f}_{\bar{j}}^{I} \operatorname{Im} N_{I J} \mathcal{F}_{0 n}^{-J}=0 .
$$


Introducing $T_{I 0 r} \equiv \operatorname{Im} N_{I J} \mathcal{F}_{0 r}^{-J}$ and separating the imaginary part of Eq.(115), we obtain

$$
k_{\bar{j} i} a_{i}^{\prime}+4 e^{-U} \operatorname{Im}\left(\bar{f}_{\bar{j}}^{I} T_{I}\right)=0
$$

Since $a_{i}$ are of the first order in string coupling, $k_{\bar{j} i}$ should be taken in the leading order. With the required accuracy, using the expressions in the section with the prepotential, we calculate

$$
\begin{aligned}
T_{00 r} & =\left[-q_{0}+i y^{3} \hat{P}^{0} \operatorname{Im} N_{00}\right] \frac{1}{2 \sqrt{-g^{\prime}}}, \\
T_{10 r} & =\left[\hat{P}^{0} \operatorname{Re} N_{10}-\hat{P}^{1}+i\left(\hat{P}^{0} \operatorname{Im} N_{10}-q_{1} \operatorname{Im} N_{11}\right)\right] \frac{1}{2 \sqrt{-g^{\prime}}}, \\
T_{20 r} & =\left[\operatorname{Re} N_{20} \hat{P}^{0}-\hat{Q}_{2}+i \operatorname{Im} N_{20} \hat{P}^{0}\right] \frac{1}{2 \sqrt{-g^{\prime}}}, \\
T_{30 r} & =\left[\operatorname{Re} N_{30} \hat{P}^{0}-\hat{Q}_{3}+i \operatorname{Im} N_{30} \hat{P}^{0}\right] \frac{1}{2 \sqrt{-g^{\prime}}} .
\end{aligned}
$$

In the combination $\operatorname{Im}\left(\bar{f}_{j}^{I} T_{I}\right)=-\operatorname{Im} f_{j}^{I} R e T_{I}+\operatorname{Re} f_{j}^{I} \operatorname{Im} T_{I}$ which enters Eq.(116) the imaginary parts of the functions $T_{I}$ with $I=1,2,3$ and $R e T_{0}$ are of the first order in string coupling. Thus, we need the corresponding functions $\operatorname{Re} f_{j}^{I}$ and $f_{j}^{0}$ in the leading order in $\epsilon$.

The functions $\operatorname{Re} f_{j}^{I}$ calculated in the leading order in $\epsilon$ are

$$
\begin{aligned}
& \operatorname{Re} f_{i}^{0}=i \frac{e^{K / 2}}{2}\left(\frac{1}{y_{1}}, \frac{1}{y_{2}}, \frac{1}{y_{3}}\right), \quad \operatorname{Re} f_{i}^{1}=\frac{e^{K / 2} y_{1}}{2}\left(\frac{1}{y_{1}},-\frac{1}{y_{2}},-\frac{1}{y_{3}}\right), \\
& \operatorname{Re} f_{i}^{2}=\frac{e^{K / 2} y_{2}}{2}\left(-\frac{1}{y_{1}}, \frac{1}{y_{2}},-\frac{1}{y_{3}}\right), \quad \operatorname{Re} f_{i}^{3}=\frac{e^{K / 2} y_{3}}{2}\left(-\frac{1}{y_{1}},-\frac{1}{y_{2}}, \frac{1}{y_{3}}\right) .
\end{aligned}
$$

Here and below $y_{i}$ are real tree-level moduli. Also we find

$$
\operatorname{Im} f_{i}^{I}=-\epsilon \frac{a_{I}}{2 y_{i}} e^{K / 2}, \quad I, i=1,2,3 .
$$

Collecting the above expressions, we obtain the system of three equations for three unknown functions $a_{i}$

$$
\begin{gathered}
a_{1}^{\prime}+\frac{4 e^{U+K / 2} y_{1}}{r^{2}}\left[q_{0}+q_{1} y_{2} y_{3}-a_{1} \hat{P}^{1}-a_{a} \hat{Q}_{a}+\hat{P}^{0}\left(-a_{1} y_{2} y_{3}+a_{2} y_{1} y_{3}+a_{3} y_{1} y_{2}\right)\right]=0 \\
a_{2}^{\prime}+\frac{4 e^{U+K / 2} y_{2}}{r^{2}}\left[q_{0}-q_{1} y_{2} y_{3}-a_{1} \hat{P}^{1}-a_{a} \hat{Q}_{a}+\hat{P}^{0}\left(a_{1} y_{2} y_{3}-a_{2} y_{1} y_{3}+a_{3} y_{1} y_{2}\right)\right]=0 \\
a_{3}^{\prime}+\frac{4 e^{U+K / 2} y_{3}}{r^{2}}\left[q_{0}-q_{1} y_{2} y_{3}-a_{1} \hat{P}^{1}-a_{a} \hat{Q}_{a}+\hat{P}^{0}\left(a_{1} y_{2} y_{3}+a_{2} y_{1} y_{3}-a_{3} y_{1} y_{2}\right)\right]=0 .
\end{gathered}
$$

Substituting the explicit tree-level expressions for the moduli and metric and using definitions (70), we obtain solution of the Eqs. (119)

$$
\begin{aligned}
& a_{1}=\frac{r}{2(P+r)}\left[c_{1}+\frac{q^{(2)}}{r}+c_{+} \ln \frac{Q+r}{P+r}-\frac{q^{(1)}}{Q} \ln \frac{r}{r+Q}+\frac{q^{(1)}}{P} \ln \frac{r}{r+P}\right], \\
& b_{2}-b_{3}=\frac{c_{-} r}{r+P}, \\
& b_{2}+b_{3}=\frac{c_{+} r+q^{(1)}}{r+Q} .
\end{aligned}
$$


Here

$$
b_{a}=\frac{a_{a}}{y_{a}} \quad q_{0}-q_{1} y_{2} y_{3} \equiv \frac{q^{(1)} e^{\gamma_{0}}}{\sqrt{8}}, \quad q_{0}+q_{1} y_{2} y_{3} \equiv \frac{q^{(2)} e^{\gamma_{0}}}{\sqrt{8}}
$$

As it was discussed in Sect.3, to have a static metric, we must take a solution for which $\operatorname{Im} T_{0 n}^{-}=0$ or $\operatorname{Re} S_{0 n}=0$ which reads

$$
2 P a_{1}+\left(b_{2}+b_{3}\right)\left(P y_{1}+Q\right)-q^{(2)}=0 .
$$

Logarithmic terms should vanish separately, thus

$$
q^{(1)}=c_{+}=0 .
$$

Thus, solution which retains the metric static is

$$
\begin{aligned}
& a_{2} e^{-\gamma_{0}-\sigma_{0}}=-a_{3} e^{-\gamma_{0}+\sigma_{0}}=c_{-} \frac{r}{r+P}, \\
& a_{1}=\frac{q^{(2)}}{2 P} .
\end{aligned}
$$

Because the axion $a_{1}$ is defined up to a constant, this means that we can consistently set $a_{1}=0$. Condition $q^{(1)}=0$ reads $q_{0}=q_{1} e^{2 \gamma_{0}}$.

General solution contains three arbitrary constants which can be adjusted so that the asymptotic ("physical") charges of the electric fields (77) and symplectic invariant $\operatorname{Im} T_{0 n}^{-}$responsible for non-stationarity of the metric vanish.

\section{Discussion}

In this paper we obtained the loop-corrected dyonic solution by solving the system of the loop-corrected Maxwell and Killing spinor equations. Loop-corrected equations of motion were derived in perturbative approach in the first order in string coupling constant from the loopcorrected $N=2$ supersymmetric effective action which has only one-loop perturbative corrections.

Except for a general theoretical interest of the study which involves a number of subtle points such as an account of ambiguities of the loop corrections to the prepotential and gauge couplings, possible modifications of the symplectic transformation connecting sections with the prepotential and associated with the heterotic string compactification, etc., explicit form of the loop corrections provide bounds on the applicability of the usually discussed tree-level solutions.

In the case of dyonic black hole with electric charges subject to the condition $\left(Q_{2} Q_{3}\right)^{1 / 2}>$ $\epsilon\left(P^{0} P^{1}\right)^{1 / 2}|h| / T U$ the loop-corrected solution (105) is valid for all $r$. For magnetic black hole with the charges $P^{0}$ and $P^{1}$ the loop-corrected solution (106) is valid for $r>\epsilon\left(P^{0} P^{1}\right)^{1 / 2}|h| / T U$ and condition of the applicability of the perturbative expansion is $r>\epsilon\left(P^{0} P^{1}\right)^{1 / 2}$.

Although the tree-level dyonic black hole is static and with real moduli, at the one-loop level the appearance of extra electric charges and imaginary parts of the moduli (axions) as well as non-stationarity parameters in the metric of the first order in string coupling is possible. 
The asymptotic (physical) charges of the extra electric field strengths depend on the axions, and by a suitable choice of free constants can be set to zero; extra magnetic charges with the accuracy of the first order in string coupling do not enter the equations of motion and can be consistently set to zero. Solving the equations for the axions, we show that, as at the tree level, there exists a stationary loop-corrected solution with four charges, but with the non-vanishing axions.

In perturbative approach, all the expressions which are of the first order in string coupling and depend on the moduli are calculated by substituting the tree-level moduli. Considerable simplifications are achieved for constant tree-level moduli $T$ and $U$. The results for dyonic black holes with four charges and full coordinate dependence of the moduli will be published elsewhere. Although the tree-level moduli are constant, the loop corrections to the moduli and the metric have full coordinate dependence.

Except for the special values of the charges $P$ and $Q$, the moduli are away from the enhanced symmetry points, where second derivatives of the prepotential have logarithmic singularities $[12,16,17]$.

The expressions for the loop corrections are valid for all $r$ for which the perturbation expansion in string coupling is valid. For the dyonic solution, the sufficient condition is $\epsilon \frac{P}{Q}<1$. In magnetic case, both the tree-level and the loop-corrected solutions can be used in the range $\frac{r}{P}>\epsilon V$. Perturbative corrections to dyonic and purely electric black holes, have finite limits $r \rightarrow 0$, in magnetic case the limit $r \rightarrow 0$ is singular.

In this paper we considered the $N=2$ supersymmetric $S T U$ model as stemming from the suitably compactified heterotic string theory. However, different embeddings of the $S T U$ model in the underlying string theory are possible $[11,31,32]$. In papers $[34,35]$ and refs. therein general classical $4 D$ BPS-saturated generating black-hole solution preserving $1 / 8$ of $N=84 D$ supergravity was constructed, and its $N S-N S$ and $R-R$ embeddings in the type IIA and IIB theories were studied. The U-duality orbit of a solution of the $N=2$ truncation of $N=8$ supergravity with $1 / 2$ supersymmetry unbroken connects solutions of type II and heterotic theories with the same amount of supersymmetry unbroken. In different embeddings of the $S T U$ model in the underlying theory the moduli and $4 D$ vector fields stem from different fields of the underlying higher-dimensional theory. Heterotic embedding, while less suitable for calculation of the microscopic entropy, is more natural for the study of stringloop corrections. In this case, dilaton is a natural string-loop expansion parameter, and the axion-dilaton parametrize a separate factor of the scalar manifold of the $S T U$ model.

String-loop corrections to the classical charged black holes as solutions of the effective $N=2$ $S T U$ model the with string-loop corrected prepotential was studied also in papers [13, 28, 36]. However, the two approaches are rather different. In these papers, the loop-corrected metric was calculated starting from the prepotential of the type II theory compactified on a CalabiYau threefold, which is dual to heterotic string compactified on $K 3 \times T^{2}$. The prepotential of type II theory is of purely classical origin, but has the same structure as the heterotic one (the prepotentials are identical under a suitable identification of the moduli in both theories). Perturbative solution was obtained under the assumption that there exists a "small" modulus on the type II side, and it is possible to expand the loop correction to the prepotential with respect to the ratios of small to large moduli. As we discussed above, within the framework 
of the heterotic string compactification on $K 3 \times T^{2}$, the natural loop-expansion parameter is expressed via the dilaton. However, this modulus does not enter the loop correction to the prepotential. The remaining moduli, which are connected to the metric and antisymmetric tensor of the compact two-torus, for special configurations may have parametric smallness, but not the functional one connected with dependence on $r$. Moreover, as we have argued above, to study the loop-corrected solution it is important to take into account corrections to the gauge couplings.

Electrically charged black holes in dilatonic Einstein-Maxwell gravity with constant threshold corrections were considered in [37]. However, the model lagrangian of this paper is different from the loop-corrected one of the present study, and hence the results are incomparable.

Solutions of the equations of motion derived from $4 D$ heterotic string effective action with $N=1$ supersymmetry with one-loop perturbative corrections (note that in this case there are contributions from all loops) and also non-perturbative corrections included were studied in [38]. However, in contrast to the present paper, only solutions with the flat space-time as well as with other simplifications were considered.

\section{Acknowledgments}

I would like to thank the members of the theoretical seminars at the Skobeltsyn Nuclear Physics Institute and Lebedev Physical Institute for discussions, M.Bertolini and M.Trigiante for useful e-mail correspondence and K. Behrndt for a remark. This work was partially supported by the RFFR grant No 02-02-16444.

\section{References}

[1] M. Green, J. Schwarz and E. Witten, Superstring Theory, (Cambridge University Press, 1987).

[2] D. Youm, Phys. Rept. 316 (1999) 1, hep-th/9710046.

[3] A.A. Tseytlin, Class. Quant. Grav. 12 (1995) 2365, hep-th/9505052.

[4] E. D'Hoker and D.H. Fong, Rev. of Mod. Phys. 60 (1988) 917.

[5] A. A. Tseytlin, Int. J. of Mod. Phys. A5 (1990) 589.

[6] I. Antoniadis, K. S. Narain and T.R. Taylor, Phys. Lett., B267, 37 (1991); I. Antoniadis, E. Gava and K. S. Narain, Nucl. Phys., B283, 93 (1992).

[7] V. S. Kaplunovsky, Nucl. Phys. B307 (1988) 145, hep-th/9205070, L. J. Dixon, V. S. Kaplunovsky and J. Louis, Nucl. Phys. B355 (1991) 649.

[8] E. Kiritsis, C. Kounnas, P.M. Petropoulos and J. Rizos, Nucl. Phys B483 (1997) 141, hep-th/9608034.

[9] E. Kiritsis, Introduction to Superstring theory, CORN-TH/97-218, hep-th/9709062. 
[10] B. de Wit and A. Van Proeyen, Nucl. Phys. B245 (1984) 89; B. de Wit, P. Lauwers and A. Van Proeyen, Nucl. Phys. B255 (1985) 569.

[11] M. J. Duff, J. T. Liu and J. Rahmfeld, Nucl. Phys. B459 (1996) 125, hep-th/9508094.

[12] B. de Wit, V. Kaplunovsky, J. Louis and D. Luest, Nucl. Phys. B451 (1995) 53, hepth/9504006.

[13] K. Behrndt et. al., Nucl. Phys. B488 (1997) 236, hep-th/9610105.

[14] M. Z. Iofa, JHEP 02 (2002) 025, hep-th/0111254.

[15] M. Z. Iofa, Mod. Phys. Lett. A17, (2002) 355, hep-th/0104163.

[16] I. Antoniadis, S. Ferrara, E. Gava, K. S. Narain and T. R. Taylor, Nucl. Phys. B447 (1995) 35, hep-th/9504034.

[17] J. A. Harvey and G. Moore, Nucl. Phys. B463, 315 (1996), hep-th/9510182.

[18] J. P. Derendinger, S. Ferrara, C. Kounnas and F. Zwirner, Nucl. Phys. B372 (1992) 145.

[19] A. Sen, Int. J. Mod. Phys. A9 (1994) 3707, hep-th/9402002.

[20] L. Andrianopoli et. al. , J. Geom. Phys. 23 (1997) 111, hep-th/9605032.

[21] A. Ceresole, R. D'Auria, S. Ferrara and A. Van Proeyen, Nucl. Phys. B444 (1995) 92, hep-th/9502072.

[22] G. L. Cardoso, B. de Wit, J. Käppeli and T. Mohaupt, JHEP 0012 (2000) 019, hepth/0009234.

[23] T. Mohaupt, Fortsch. Phys. 49 (2001) 3, hep-th/0009234.

[24] E. Fradkin and A. Tseytlin, Phys. Lett. B158 (1985) 316; A.A. Tseytlin, Phys. Lett. B223 (1989) 165.

[25] L. Castellani, R. D’ Auria and S. Ferrara, Phys. Lett. B241 (1990) 57.

[26] R. D'Auria, S. Ferrara and P. Fre, Nucl. Phys. B359 (1991) 705.

[27] S. Ferrara and R. Kallosh, Phys. Rev. D54 (1996) 1514, hep-th/9602136.

[28] K. Behrndt, D. Lust and W. A. Sabra, Nucl. Phys. B510 (1998) 266, hep-th/9705169.

[29] P. Fre, Nucl. Phys. Proc. Suppl. 57 (1997) 52, hep-th/9701054.

[30] M. Z. Iofa, Phys. Lett. B, to appear, hep-th/0204093.

[31] M. Cvetič and D. Youm, Nucl. Phys. B453 (1995) 259, hep-th/9505045; Phys. Rev. D53 (1996) 584, hep-th/9507090. 
[32] M. Cvetič and A. A. Tseytlin, Phys. Lett. B366 (1996) 95, hep-th/9510097; Phys. Rev. D53 (1996) 5619, hep-th/9512031.

[33] G. W. Gibbons and C. M. Hull, Phys. Lett. B109 (1982) 190.

[34] M. Cvetič and C. M. Hull, Nucl.Phys. B480 (1996) 296, hep-th/9606193.

[35] L. Andrianopoli et.al., Nucl.Phys. B509 (1998) 463, hep-th/9707087; M. Bertolini, P. Fre and M. Trigiante, Class. Quant. Grav. 16 (1999) 1519, hep-th/9811251; M. Bertolini and M. Trigiante, Int. J. Mod. Phys. A15 (2000) 5017, hep-th/9910237; Nucl. Phys. B582 (2000) 393, hep-th/0002191.

[36] K. Behrndt and I. Gaida, Phys. Lett. B401, 263 (1997), hep-th/9702168; K. Behrndt, G. Lopes Cardoso and I. Gaida, Nucl. Phys.B506, 267 (1997), hep-th/9704095.

[37] K.-L. Chan, Mod. Phys. Lett. A12 (1997) 1597, hep-th/9610121.

[38] A. A. Tseytlin, hep-th/9402082; M. Cvetič and A. A. Tseytlin, Nucl. Phys. B416 (1994) 137, hep-th/9307123. 